\title{
Blind Identification for Communication Signals: An Approach Exploiting Sampling Phase Diversity
}

\author{
Emmanuel Racine, Dominic Grenier \\ Laboratoire de Radiocommunications et de Traitement du Signal, Laval University, Canada \\ Correspondence: Emmanuel Racine, emmanuel.racine.2@ulaval.ca \\ Manuscript communication: received 5 December 2012, accepted 18 March 2013
}

\begin{abstract}
This paper presents a second-order blind channel estimation algorithm for digital communication signals under natural asynchronous conditions where no synchronization exists between transmitting and receiving antennas. The approach exploits sampling phase diversity provided by the cyclostationary nature of the received signals as an advantageous means of constructing multiple sets of distinct autocorrelation matrices evaluated at equals time lags. Channel estimation is then performed via joint diagonalization of a set of differential autocorrelation target matrices, avoiding the need of noise power or statistical distribution estimation. A broad set of simulation experiments is presented in distinctive signal contexts as a means of supporting and evidencing the potential of the proposed estimation method.
\end{abstract}

Keywords- Array processing, signal model, blind identification, blind source separation, joint diagonalization, approximate joint diagonalization, cyclostationarity, synchronous signals, asynchronous signals.

\section{INTRODUCTION}

Array processing techniques for communication signals have received considerable amount of attention over the past decades. The potential advantages of such techniques in communication systems have been widely discussed in numerous books and papers [14] and are well known to the researchers in the field. Consistently, significant efforts have been invested in the development of parameter estimation algorithms which are expected to go hand-in-hand with future generations of wireless communication systems. Ranging from classical second-order algorithms [5, 6] to higher order cumulant-based approaches [7-10], encompassing spectral and non-linear statistical theories [11-15], the literature is filled with a diversity of estimation algorithms exploiting various statistical signal properties.

Particularly, the general blind source separation (BSS) problem has received significant attention since the contributions of JADE [16] and subsequently SOBI [17], where in the latter case a statistically more attractive approach exploiting second-order autocorrelation of the received signals at different time lags was proposed. The source signals and mixing matrix were then estimated after joint diagonalization (JD) of a set of resulting target matrices. This underlying philosophy has been a source of inspiration for many works to follow [18-22], and will also be exploited in this paper.

JADE-like algorithms also perform blind estimation from the joint diagonalization of a set of target matrices, which are however constructed from the higherorder moments of the received signals. These two types of similar processing techniques have given rise and motivated the development of high-performance JD algorithms to the point of becoming a research topic of its own [22-30] with particular dedication to the BSS problem.

In this paper, we develop a blind channel estimation algorithm for digital communication signals following the same philosophy. The procedure will basically consist of generating a set of Hermitian target matrices from second-order expectations of the received signals, then obtain an estimate of the mixing (or channel) matrix by solving an approximate joint diagonalization problem (AJD). The latter problem arises when considering non-ideal target matrices such as those obtained from a finite number of samples. A fundamental difference in our approach however is that instead of simply considering autocorrelation evaluations at different values of time lag as in [17, 19-21], we show that the cyclostationary nature of the received signals under natural asynchronous conditions can also be exploited to obtain similar statistical expectations at different time values, therefore providing additional autocorrelation matrices for the AJD problem. The operation is numerically achieved by computing statistics from multiple sets of baud-sampled sequences in an interleaved fashion, and is referred to as exploitation of sampling phase diversity.

The joint use of both time and delay for autocorrelation matrix computation in JD-based estimation algorithms has already been proposed in the literature, for example in the case of nonstationary source separation using simultaneous diagonalisation (NSS-SD) [31-33]. However, instead of solving the AJD problem considering the immediate set of autocorrelation matrices obtained as a preliminary step, we propose the construction of target matrices based on a differential formula 
between autocorrelation matrices evaluated at identical time lags. This approach, termed JDDTM referring to joint diagonalization of differential target matrices (DTM), offers various advantages such as robustness vis-à-vis noise statistical distributions as well as full exploitation of the array's degree of freedom. Details of the process will be further explained in Section 4 . The latter approach for the construction of target matrices from exploitation of sampling phase diversity provided by the cyclostationary nature of communication signals in a general asynchronous context is the main contribution of the paper.

An interesting contrast is to be made between our said estimation approach and the work of Rong et al. in [18], which in some way has been developed in a similar practical context. The proposed approach is highly inspired from [17] and [34], with the fundamental difference of using only zero-lag autocorrelation matrices obtained by a controlled time-varying power loading scheme where users' transmit power is varied to provide the necessary autocorrelation diversity required for application of a JD process. The algorithm is efficient in both synchronous and asynchronous cases, and also applies to identically modulated signals. However, it does require a certain amount of coordination between user mobiles and the receiving array in order to correctly gauge the time intervals over which power is varied and estimation of the subblock autocorrelation matrices is performed. We wish to point out that one of the main objective of this paper was to develop a comparable blind estimation algorithm requiring no such synchronisation mechanism between emitting and receiving antennas. The necessary autocorrelation diversity is here obtained by direct exploitation of the cyclostationarity nature of communication signals under natural asynchronous propagation. It will be furthermore shown that variable power loading effects similar to those considered in [18] can be obtained in both synchronous and asynchronous cases by simply varying the sampling phase at which a zero-lag autocorrelation matrix is computed, without requiring true transmit power variation on the uplink.

A particular emphasis is brought on signal modeling in Section 2, especially for clearly evidencing the differences between synchronous and asynchronous signal models and their respective implications on the discrete-time expression of the received signal vector. Section 3 presents the general autocorrelation matrices construction principle based on exploitation of sampling phase diversity while Section 4 is concerned with the JD problem itself. The description of the DTM construction principle will also be presented therein, along with two distinct resolution approaches considering either the full or a restricted set of target matrices. Finally, Section 5 presents a wide range of simulation results and analysis under various signal conditions, including a comparative study of performance considering different AJD algorithms and a behavioural examination of performance under worst-case/unfavorable estimation conditions.

Throughout the paper, '*', ' $\dagger$ ', ' $\top$ ' and '\#' are re- spectively used to denote the conjugate, the conjugate transpose, the transpose and the Moore-Penrose pseudoinverse operator. An element $p$ of a vector $a$ will be represented by either $a_{p}$ or $[a]_{p}$ and likewise an element $p q$ of a matrix $A$ will be represented by $A_{p q}$ or $[A]_{p q}$.

\section{Signal Modeling}

\subsection{The Received Signal Vector}

We consider a traditional uplink scenario in which a number of $M$ co-channel signals impinge on a $N$ element antenna array of arbitrary geometry. The timecontinuous received signal vector is given by:

$$
x(t)=A s(t)+n(t),
$$

where $A \in \mathbb{C}^{N \times M}$ is the array manifold matrix, $s(t) \in$ $\mathbb{C}^{M \times 1}$ is the received baseband source vector and $\boldsymbol{n}(t)$ is a general additive noise assumed stationary. Each element $x_{i}(t)$ of $x(t)$ is measured as the output of a quadrature demodulator [35] assuming ideal carrier phase recovery ${ }^{1}$. Note that eq. (1) implicitly assumes narrowband signal propagation with respect to the array dimensions.

As in [18], we consider a propagation environment in which frequency-selective effects (or delay spread) are negligible. This assumption is of crucial importance in order to obtain autocorrelation matrices having the necessary eigenstructure for a JD-based estimation approach. Assuming that communication is established with $G$ independent users during a given observation time, the $M \times 1$ vector $s(t)$ can be linked with a $G \times 1$ received baseband source signal vector $\boldsymbol{u}(t)$ for all $G$ users such that:

$$
\begin{aligned}
s(t)=\left[\begin{array}{c}
s_{1}(t) \\
s_{2}(t) \\
\vdots \\
s_{G}(t)
\end{array}\right] & =\left[\begin{array}{cccc}
\alpha_{1} & \mathbf{0} & \ldots & \mathbf{0} \\
\mathbf{0} & \boldsymbol{\alpha}_{2} & \ldots & \mathbf{0} \\
\vdots & \vdots & & \vdots \\
\mathbf{0} & \mathbf{0} & \ldots & \boldsymbol{\alpha}_{G}
\end{array}\right]\left[\begin{array}{c}
u_{1}(t) \\
u_{2}(t) \\
\vdots \\
u_{G}(t)
\end{array}\right] \\
& \equiv \boldsymbol{\Xi} \boldsymbol{u}(t),
\end{aligned}
$$

where $\left\{\boldsymbol{\alpha}_{g} \in \mathbb{C}^{M_{g} \times 1}\right\}_{g=1}^{G}$ are the complex multipath coefficient vectors of the $G$ coherent groups of signals (a $g$-th group is comprised of the $M_{g}$ impinging signals from user $g$ such that $\sum_{g=1}^{G} M_{g}=M$ ), and where $\left\{u_{g}(t)\right\}_{g=1}^{G}$ are the corresponding received elementary baseband source signals assumed uncorrelated with each other, i.e. $E\left\{u_{p}(t) u_{q}(t)\right\}=\sigma_{u_{p}}^{2}(t) \delta_{p, q}$ where $\delta_{p, q}$ is the Kronecker delta. Substituting (3) into (1) yields:

$$
\boldsymbol{x}(t)=A \Xi \boldsymbol{u}(t)+\boldsymbol{n}(t) \equiv \boldsymbol{B} \boldsymbol{u}(t)+\boldsymbol{n}(t),
$$

${ }^{1}$ Note that the notion of carrier phase recovery makes no particular sense in this context since the instantaneous carrier phase values of each impinging co-channel RF signals (from either independent users or reflected paths in the environment) at a precise receiving antenna location will generally not be identical. Consequently, a local oscillator (LO) may lock with at most one of these impinging carriers which would have to be tracked or distinguished from the received RF mixture. Achieving carrier frequency recovery on the other hand is a sufficient condition to ensure validation of eq. (1), where phase differences between RF signals and the LO simply induce fixed constellation rotations that can be absorbed in either $A$ or $s(t)$. 
where $B=A \Xi$ is called the generalized steering matrix (GSM) of the sources which, in addition to complex multipath coefficients, may also encompass the effects of amplitude and phase mismatches as well as mutual coupling between array elements. A model identical to (4) has also been considered in [36, 37], and will be used throughout the rest of the paper. The discrete-time equivalent of (4) is given by:

$$
\boldsymbol{x}_{k}=\boldsymbol{B} \boldsymbol{u}_{k}+\boldsymbol{n}_{k}
$$

where index $k$ denotes a measurement taken at time $t=t_{k}=k T_{s}$ where $T_{s}$ is the sampling period. Blind identification refers to the estimation of $\boldsymbol{B}$ given sole observation of vectors $\left\{\boldsymbol{x}_{k}\right\}_{k=1}^{K}$.

\subsection{The Received Source Signals}

2.2.1 Synchronous and Asynchronous Signal Modeling: The fundamental difference between the two types of modeling lies in the interpretation of the source signals $u_{g}(t)$ in (4). The literature sometimes provides misleading or at least different definitions of the appellation depending on the problem at hand. For example, in [38], synchronisation is achieved when the received source signals have zero delay of arrival. In [39, 40], the same terminology refers to symbol timing control in microcell applications, and in [18], it refers to the boundaries of epochs over which user transmit powers are kept constant, with no regard to symbol timing.

Although these definitions are correctly adapted to the context of their respective problem, we aim in this section at giving a clearer understanding of the two types of signal models based on symbol as well as sampling timing considerations, which are shown to have a more profound impact on the statistical distribution of $x_{k}$ in (5). Considering the case of generic QAMmodulated signals, a complex envelope $u_{g}(t)$ can be expressed as [39, 41, 42]:

$$
u_{g}(t)=\sum_{m} s_{g_{m}} p_{g}\left(t-\tau_{g}-m T\right),
$$

where $\left\{s_{g_{m}}\right\}_{m}$ and $p_{g}(t)$ are the zero-mean iid transmitted symbol sequence of a $g$-th user and its corresponding received pulse shaping function. Parameter $T$ represents the symbol period assumed identical for all users. We refer to $\tau_{g}$ in (6) as the asynchronous delay of the $g$-th user, and the sources are said to be baudsynchronized if $\tau_{g}=\tau_{0} \forall g$.

Fig. 1 displays a comparison between synchronous and asynchronous signals. For simplicity, $G=2$ users are considered with BPSK modulations and raised cosine (RC) impulse functions resulting from ideal matched filtering. The source signals are plotted in each case to better draw correspondences with the output signal $x_{i}(t)$, where $i$ is the index of an arbitrary element of the array, but are otherwise unobservable in a practical context. Fig. 1 as well as autocorrelation matrices $\boldsymbol{R}_{x x_{1}}, \boldsymbol{R}_{x x_{1}}^{\prime}$ and $\boldsymbol{R}_{x x_{2}}^{\prime}$ will also serve as a reference in Section 3 to detail the computation process of autocorrelation matrices in a more intuitive way. This section will only be concerned with the implications of both modelings in eq. (5).
Fig. 1 (a) depicts the case of synchronous signals. The symbol instants of all source signals are precisely coincident due to the equality $\tau_{1}=\tau_{2}$ in (6). This modeling has been and is still extensively used in the literature, and can be especially noticed for algorithms in which a numerical implementation of (5) is such that elements of vector $\boldsymbol{u}_{k}$ (or any equivalent received source signal vector) are given values at each time $t_{k}$ among sets of ideally transmitted symbols (in the case where $T_{S}=T$ ). For example, this approach has been particularly popular in BSS algorithms exploiting higherorder statistics (HOS) [7-10, 36, 43] of the received signals. It is also encountered in estimation theories exploiting non-linear operators such as the sources' characteristic function [13-15], and more importantly in algorithms relying on the finite alphabet (FA) property of the source signals $[44-48]^{2}$. In the latter case, the digital nature of the transmitted signals is efficiently exploited by noting that samples associated with each source are restricted to a finite alphabet. Consequently, elements of the received signal vector are also limited to a finite number of possible values (neglecting noise). The situation is clearly evidenced in Fig. 1 (a) where at each sampling instant, signal $x_{i}(t)$ takes only four different values in either the I or Q branches, resulting from the summation of two binary random variables. Considering noise, four clusters would hence be produced in the complex plane of this $i$-th element from which center locations can be estimated and used to general estimation purposes.

From a practical point of view, it follows that the synchronous signal assumption which allows a convenient replacement of the source signal values at each sampling instant by a set of ideally transmitted symbols (for $T_{s}=T$ ) can only be valid if

1) Symbol clock phase can be precisely controlled for each user such that baud synchronicity is achieved for all impinging signals after down-converison and filtering at the antenna array ${ }^{3}$. Such a control can only be achieved using a synchronisation reference signal generated either from the base station (presumably), within mobile users themselves or from an external source (e.g. satellite clocks [49]).

2) Accurate symbol timing recovery is performed at the receiving array from the sole observation of $x(t)$ and/or the help of a synchronisation reference signal.

On the other hand, Fig. 1 (b) illustrates the case of asynchronous signals considering the same channel coefficients and transmitted symbol sequences as in (a). A sampling period of $T_{S}=T / 2$ is now considered but is only intended to better introduce the theory of Section 3. It can be observed that the symbol instants of the the source signals are now no longer coincident,

\footnotetext{
${ }^{2}$ References [7-10, 36, 43], [13-15] and [44-48] have been specially selected such that the reader finds explicit evidence of a numerical implementation of (5) in which elements of $\boldsymbol{u}_{k}$ are given values among ideal sets of transmitted symbols.

${ }^{3}$ From a limited distance of the array and under negligible delay spread, such an operation can be achieved if the transmitters have identical symbol clock phases.
} 
(a)

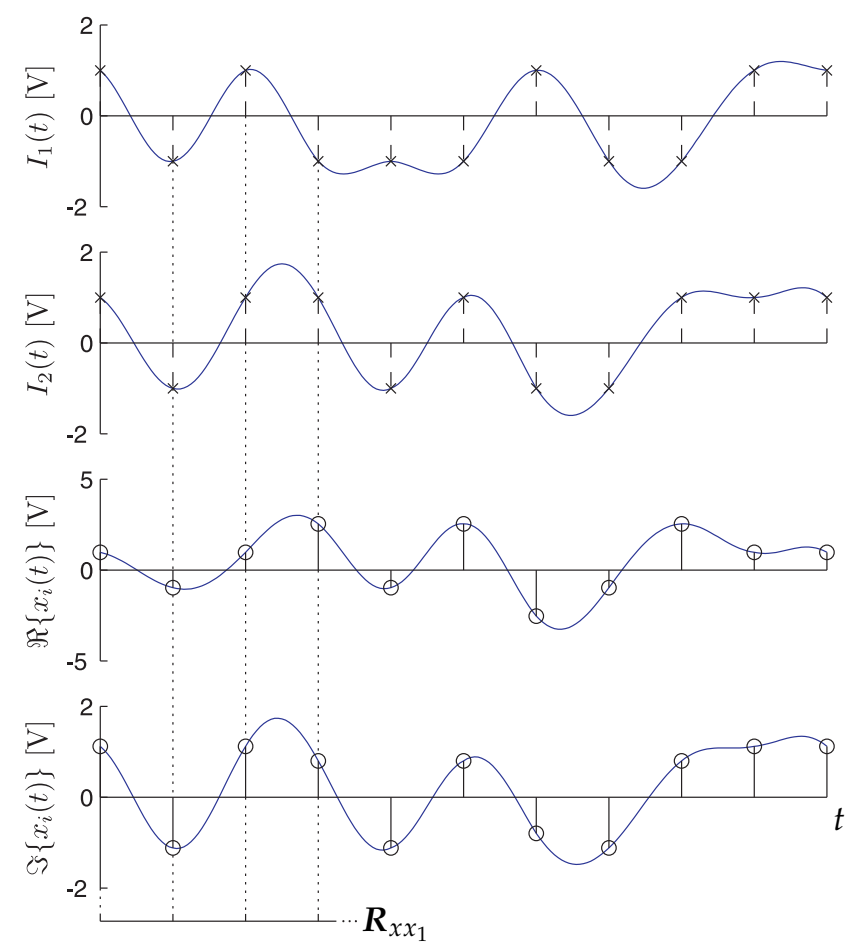

(b)

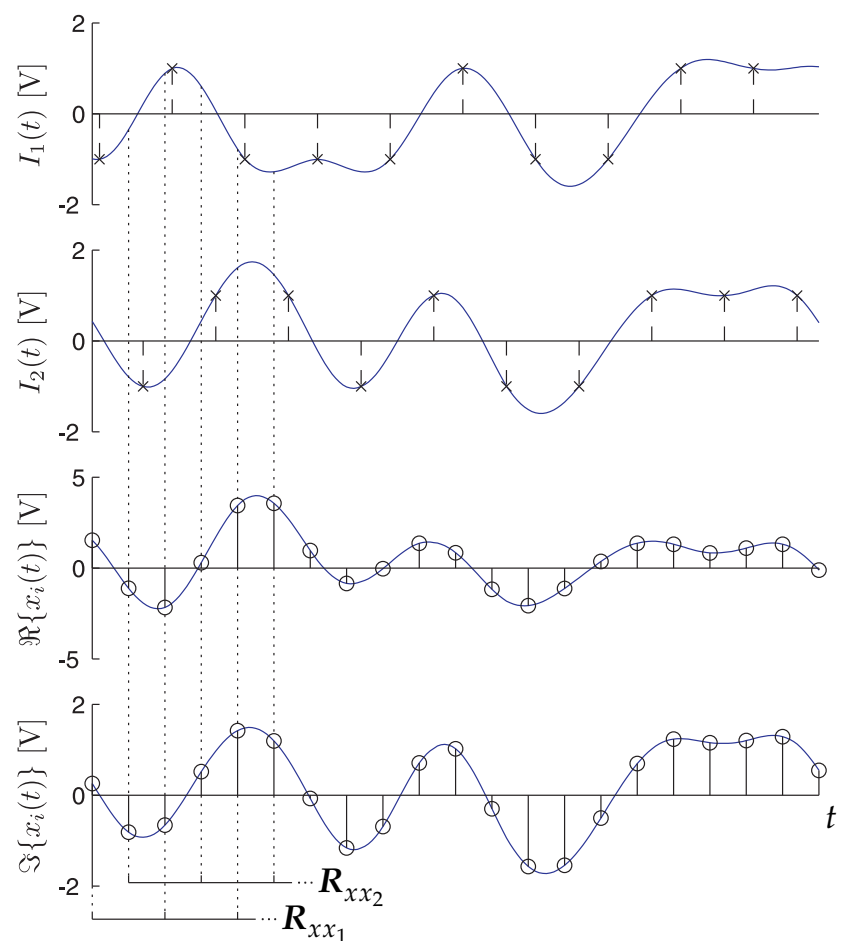

Figure 1. Comparison between synchronous (a) and asynchronous (b) signal modeling, and illustration of source power variation obtained by sampling phases diversity (b). The noiseless received signal on a given $i$-th element is represented considering two BPSK sources with channel coefficients $B_{i 1}=-0.8 e^{-j 0.2}$ and $B_{i 2}=2 e^{j 0.5}$. A source signal is represented by $u_{g}(t)=I_{g}(t)+j Q_{g}(t)$ where $Q_{g}(t)$ is set to 0 in this particular example.

which results from the condition $\tau_{1} \neq \tau_{2}$ in (6). A parameter $\tau_{g}$ has been introduced in (6) as the asynchronous delay of a user $g$. However it is not to be confused with the actual time of arrival of a given signal to the receiver. For example, consider that sources $u_{1}(t)$ and $u_{2}(t)$ in Fig. 1 (b) correspond to complex envelopes of two distinct line-of-sight (LOS) signals from users located at an equal distance of the array. In such a situation, the received signals have identical time of arrivals. However, one may observe that $\tau_{1} \neq \tau_{2}$ if user symbol clocks have different phases. Conversely, received signals from two users located at different distances from the array (i.e. having different times of arrivals) may also be such that $\tau_{1}=\tau_{2}$ if appropriate conditions on symbol clock phases are satisfied (e.g. achieving synchronisation as in Fig. 1 (a)). A parameter $\tau_{g}$ in (6) may therefore be interpreted as encompassing the effects of both signal time of arrival and symbol clock phase. We may impose:

$$
\tau_{g} \in[0, T[\forall g \in\{1,2, \ldots, G\},
$$

which is a restriction of sufficient flexibility to characterize the asynchronous nature of the sources given the statistical periodicity of (6) for minimal lengths of transmitted symbol sequences. Note that $\tau_{g}$ is defined with respect to the first sampling instant $(t=0)$ of $x(t)$, consistently with Fig. 1 . A value of $\tau_{g}=0$ hence implies that the symbol instants of a $g$-th source signal are coincident with the sampling instants (in the specific case where $T_{S}=T$ ).
The situation of Fig. 1 (b) is to be naturally expected in a co-channel communication system where independent users are likely to transmit data at arbitrary times from each other in an asynchronous fashion. Although the case of only two users is considered for simplicity, extension of the same principles apply to any number of impinging source signals. Even if $T_{S}=T$, it is important to note that under asynchronous conditions sampling instants of an observable signal $x_{i}(t)$ will generally not coincide with the symbol instants of the sources $^{4}$. Consequently elements of vector $\boldsymbol{u}_{k}$ in (5) cannot be given values among ideal sets of transmitted symbols at each discrete time $t_{k}$. The statistical distribution of $u_{g}\left(t_{k}\right)$ strongly depends on $\tau_{g}$, and to better illustrate this dependency, Fig. 2 displays two scatter plots of $x_{i}\left(t_{k}\right)$ from Fig. 1 considering both synchronous and asynchronous modelings, where in the latter case two different sets of asynchronous delays are considered. With the addition of noise, the clustering nature of $x_{i}\left(t_{k}\right)$ is easily observed in (a). The property is quickly lost however in (b) as $\tau_{1} \neq \tau_{2} \neq 0$.

2.2.2 FIR-MIMO Modeling: Asynchronous signals can be numerically modeled using (5) where elements of $\boldsymbol{u}_{k}$ are given values according to (6) for any $t=t_{k}$. Alternatively, $x_{k}$ may also be expressed using the well known finite impulse response multiple-input multipleoutput (FIR-MIMO) signal model which is extensively used in blind identification problems involving both

\footnotetext{
${ }^{4}$ This could also be the case for synchronous signals if $\tau_{g}=\tau_{0} \neq$ $0 \forall g \in\{1,2, \ldots, G\}$.
} 


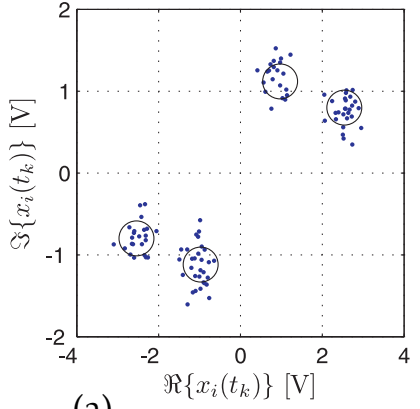

(a)

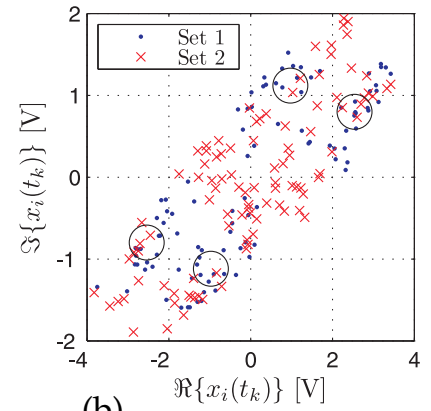

(b)
Figure 2. Scatter plots of an observable signal $x_{i}(t)$ sampled at the symbol rate considering synchronous (a) and asynchronous (b) source signals. Parameters identical to those of Fig. 1 are considered, with the addition of a noise of identical power in both cases. Asynchronous signals are modeled in (b) considering set $1:\left\{\tau_{1}=0.1 T, \tau_{2}=0.2 T\right\}$ and set 2: $\left\{\tau_{1}=0.9 T, \tau_{2}=0.5 T\right\}$. Theoretical exact cluster center locations are identified by black circles in (a) and reported in (b) for comparison.

synchronous or asynchronous signals. We will now perform a brief review of the general underlying principles of this model in order to compare the advantages and disadvantages of the two types of modeling for the problem at hand. At the same time, we also wish to bridge a gap between the somewhat different mentalities existing within classical array processing and general MIMO systems concepts.

For convenience, we first consider the case of a single user, single transmit antenna and multiple receive antennas (SU-SIMO). The received signals for an uplink transmission considering a FIR signal model can be expressed at a time $t_{k}=t_{0}+k T$ such that:

$$
\boldsymbol{x}_{k}=\boldsymbol{H}_{1} \boldsymbol{s}_{1_{k}}+\boldsymbol{n}_{k}
$$

where $\boldsymbol{H}_{1} \in \mathbb{C}^{N \times L_{1}}$ is a symbol response channel matrix and $s_{1_{k}} \in \mathbb{C}^{L_{1} \times 1}$ is a vector of $L_{1}$ consecutive transmitted symbols (see [41], eq. (17)). Vectors $x_{k}$ and $n_{k}$ are the same as in (5). The model of eq. (8) is not limited to zero delay spread (as it is (5), but not necessarily in (1)), and represents a convenient way of expressing the received signals in terms of the transmitted symbols of a particular user, or source, which are often the sole parameters of interest in a communication link. Matrix $H_{1}$ accounts for both the pulse shaping waveform $p_{1}(t)$ and the sampling phase (accounted for by $t_{0}$ ) of the received signal, whereas these parameters are directly encompassed in $\boldsymbol{u}_{k}$ in (5). Neglecting noise, the correspondence between (5) (for a single user) and (8) is such that:

$$
\begin{aligned}
& \boldsymbol{x}_{k}=\boldsymbol{B} u_{1}\left(t_{k}\right)=\boldsymbol{H}_{1} s_{1_{k}}
\end{aligned}
$$

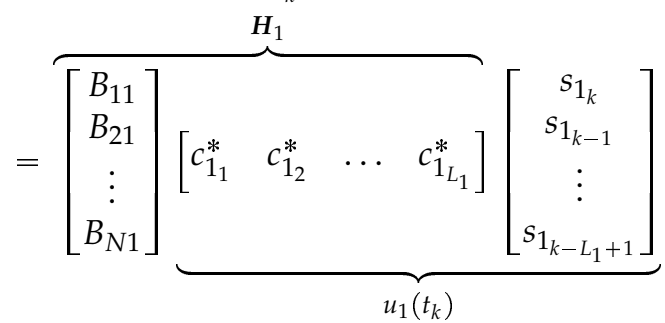

$$
\begin{aligned}
& \equiv \boldsymbol{b}_{1} \boldsymbol{c}_{1}^{\dagger} \boldsymbol{s}_{1_{k}}
\end{aligned}
$$

where coefficients $\left\{c_{p}\right\}_{p=1}^{L_{1}}$ are such that $c_{1}^{\dagger} s_{1_{k}}=u_{1}\left(t_{k}\right)$ for a given $t_{0}$. This relation is a simple means of expressing the value of an analogue source sample $u_{1}\left(t_{k}\right)$ in terms of a weighted sum of consecutively transmitted symbols, where the number of coefficients (or dimension of $\left.s_{1_{k}}\right), L_{1}$, is known as the channel memory, or length. For example, in the case of Fig. 1 (a), sampling and symbol instants of the sources are coincident, and $\mathrm{RC}$ pulses respect the Nyquist criterion (no intersymbol interference (ISI) at the sampling instants). Therefore, for any of the two signals (say $u_{1}(t)$, assuming that $\left.u_{2}(t)=0\right), L_{1}=c_{1}=1$, implying that $x_{i}\left(t_{k}\right)=$ $B_{i 1} s_{1_{k}}$. Conversely, still in the case of Fig. 1 (a) but considering a different sampling phase, or in a general asynchronous condition such as Fig. 1 (b), sampling instants do no longer coincide with symbol instants of the sources. Hence, $x_{i}\left(t_{k}\right)=\boldsymbol{b}_{1} \boldsymbol{c}_{1}^{\dagger} \boldsymbol{s}_{1_{k}}$ (neglecting $u_{2}(t)$ ) with $c_{1}$ depending on the sampling phase and the nature of the received pulse waveform $p_{1}(t)$, and $L_{1}$ of time width the latter. The explicit dependency of $c_{1}$ on $t_{0}$ has been dropped in (9) and (10) for clarity.

We proceeded to this detailed description of the SUSIMO signal model in order to underline an important point regarding the theoretical exact value of $L_{1}$. In the literature, channel memory is often solely related to the delay spread relative to the symbol period of a given signal. For example, Glisic in [50] (eq. (6.27)) derives a simple relation in this regard:

$$
L_{1}=\left[\frac{T_{\max _{1}}}{T}\right]+1,
$$

where $T_{\max _{1}}$ is the maximum delay spread among all multipaths (still considering a single user), and [.] is the integer part function. Examples of recent works considering a similar definition can also be found in [51-53]. It follows from (11) that for $T_{\max _{1}}=0$ (e.g. a direct LOS signal), $L_{1}=1$, which corresponds to the situation of Fig. 1 (a). However, for any different sampling phase or under asynchronous conditions, the latter equality no longer holds since energy from more than one symbol will contribute to the value of the considered source signal at all sampling instants. Eq. (11) correctly predicts the memory induced by the channel due to physical propagation (i.e. delay spread), but neglects the memory inherent to the pulse-shaped nature of the source signal itself which is directly involved in (10). Fig. 3 shows that for a pulse shaping function $p_{g}(t)$ of width $W_{g}$, up to $\left\lceil W_{g} / T\right\rceil$ consecutive symbols contribute to the value of $u_{g}(t)$ in (6) at any time $t$. Therefore, a minimum and sufficient value of $L_{1}$ may be obtained by rewritting (11) such that:

$$
L_{1}=\left\lceil\frac{T_{\max _{1}}}{T}\right\rceil+\left\lceil\frac{W_{1}}{T}\right\rceil .
$$

The total signal memory to be used in (10) for accurate modeling of the received signal possesses an upper bound depending on the maximum delay spread, but also on the particular shape of the received symbol pulses at the receiver. Note in general that the representation of $s_{1}\left(t_{k}\right)$ in (9) is not rigorously exact considering 


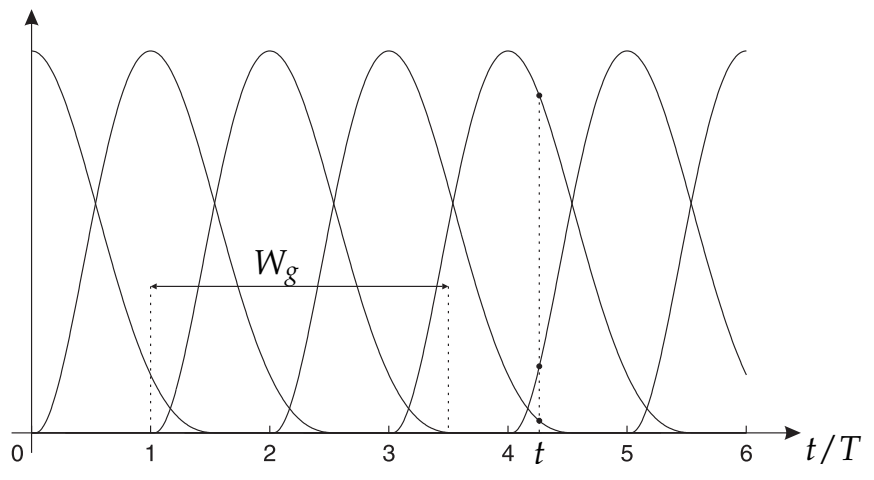

Figure 3. Pulses of an arbitrary pulse-shaped received signal $u_{g}(t)$ showing the contribution of consecutive symbols to the signal value at a time $t$. This example is for a pulse width of $W_{g}=2.5 T$.

that the value of a given source signal does not only depend on past but also on future symbols to be received (see Fig. 3). Nonetheless, $s_{1}\left(t_{k}\right)$ would still form a set of $L_{1}$ consecutively transmitted symbols and one could always consider a rectified representation of (8) by replacing $x_{k}$ with an appropriate time-delayed version of itself such that $\left[s_{1_{k}}\right]_{1}=s_{1_{k}}$ in (9). It is interesting to note that given the fact that practical pulse shaping filters are implemented considering impulse responses having typical lengths of up to 12 symbol periods [54], channel memory due to the ISI nature of a source signal itself may account for a larger fraction of the total signal memory than its counterpart depending on the maximum delay spread. From such considerations, it also follows that the assumption of a memoryless channel $\left(L_{1}=1\right)$ can only hold under the synchronous case of Fig. 1 (a), or if $W \leqslant T$.

Extension of (8) to the multiple users case can be performed by first expressing vector $\boldsymbol{u}_{k}$ from analogy with (9) such that:

$$
\begin{aligned}
{\left[\begin{array}{c}
u_{1}\left(t_{k}\right) \\
u_{2}\left(t_{k}\right) \\
\vdots \\
u_{G}\left(t_{k}\right)
\end{array}\right] } & =\left[\begin{array}{cccc}
c_{1}^{\dagger}\left(t_{0}\right) & \mathbf{0} & \ldots & \mathbf{0} \\
\mathbf{0} & c_{2}^{\dagger}\left(t_{0}\right) & \ldots & \mathbf{0} \\
\vdots & \vdots & & \vdots \\
\mathbf{0} & \mathbf{0} & \ldots & c_{G}^{\dagger}\left(t_{0}\right)
\end{array}\right]\left[\begin{array}{c}
\boldsymbol{s}_{1_{k}} \\
\boldsymbol{s}_{2_{k}} \\
\vdots \\
\boldsymbol{s}_{G_{k}}
\end{array}\right] \\
& \equiv C\left(t_{0}\right) s_{k},
\end{aligned}
$$

where $\boldsymbol{c}_{g}\left(t_{0}\right) \in \mathbb{C}^{L_{g} \times 1} \forall g$, and where $\boldsymbol{s}_{k} \in \mathbb{C}^{\left(\sum_{g} L_{g}\right) \times 1}$ is the concatenation of all users' transmitted symbol sequences. The channel length $L_{g}$ for each user is obtained by (12) with substitution of an appropriate index. The received signal vector is then given by:

$$
\boldsymbol{x}_{k}=\overbrace{\boldsymbol{B} \underbrace{\boldsymbol{H}\left(t_{0}\right)}_{\boldsymbol{u}\left(t_{k}\right)} \boldsymbol{s}_{k}}^{\boldsymbol{H}\left(t_{0}\right)}+\boldsymbol{n}_{k},
$$

with

$$
\begin{gathered}
\boldsymbol{H}\left(t_{0}\right)=\left[\begin{array}{cccc}
\boldsymbol{H}_{1}\left(t_{0}\right) & \boldsymbol{H}_{2}\left(t_{0}\right) & \ldots & \boldsymbol{H}_{G}\left(t_{0}\right)
\end{array}\right], \\
\boldsymbol{H}_{g}\left(t_{0}\right)=\boldsymbol{b}_{g} \boldsymbol{c}_{g}^{\dagger}\left(t_{0}\right) .
\end{gathered}
$$

Note that despite their similar notations, $s_{k}$ in (14) is not to be confused with $s\left(t_{k}\right)$ in (2). The general structure of the FIR-MIMO signal model of eq. (14) is valid for any delay spread under narrowband propagation and time-invariant channel assumptions, but is specific for a sample period of $T_{S}=T$. Modeling for higher sampling rates is possible as explained in [41] (Fig. 7). Considering an integer oversampling factor $U$ such that $T_{S}=T / U$, the set of all samples can be viewed as $U$ distinct baud-sampled sequences having different sampling phase $t_{0}$. Hence one obtains $U$ matrix equations akin to (14) of the type $\boldsymbol{x}_{k}^{(u)}=\boldsymbol{H}\left(t_{0}^{(u)}\right) \boldsymbol{s}_{k}+\boldsymbol{n}_{k}^{(u)}$ for $u \in\{1,2, \ldots, U\}$ that can be used for subsequent processing ${ }^{5}$.

The second and more important point we wish to highlight in this analysis is related to the differences in using either of the two models in eq. (14) under oversampling conditions. The use of a FIR-MIMO signal model is a convenient way of expressing the observable outputs in terms of the transmitted symbol sequences of each user, but implies having to deal with a different channel matrix for each of the $U$ sampling phases considered. On the other hand, the use of the classical model (5) allows the received signals to be expressed in terms of the complex envelopes of the sources, lacking an explicit reference to the transmitted symbols, but implies that matrix $\boldsymbol{B}$ to remains constant independently of the sampling phase since the latter is absorbed in $\boldsymbol{u}_{k}$ (the dependency of $\boldsymbol{u}_{k}$ on $t_{0}$ has been omitted in (14) for brevity). This property will prove particularly useful for the problem at hand considering an estimation of the channel matrix obtained via JD where target matrices have to share a congruent form (e.g. see [24, 26]). For this reason, a modeling of $x_{k}$ according to (5) will be retained for the rest of the paper instead of a FIR-MIMO equivalent in (14).

\section{Exploiting Sampling Phase Diversity}

This section describes how sampling phase diversity combined with the cyclostationarity nature of the received signals under natural asynchronous conditions can be exploited to construct a set of autocorrelation matrices suitable for a general BSS problem. Details about the specific JD operation for estimation of $\boldsymbol{B}$ will be given in Section 4 .

As mentioned in the introductory section of the paper, the work of Rong et al. in [18] has also been developed in a context of wireless communications, where estimation of the channel matrix was performed considering a set of zero-lag autocorrelation matrices. Prior to undergoing any further analysis related to the current problem, we first wish to rectify a number of claims made by the same authors regarding some aspects of signal apprehension in a general wireless communication context.

On page 2, the authors advocate the use of zerolag autocorrelation matrices by describing some limitations inherent to SOBI-like algorithms where multiple autocorrelation evaluations of the received signals at

\footnotetext{
${ }^{5}$ Note that vector $s_{k}$ remains constant for each sample sequence since data values are also constant over one symbol period [41].
} 
non-zero lags are exploited. It is first claimed that application of the latter methods requires the existence of a long-time coherence of the source signals in order to obtain enough cross-covariance matrices for a joint diagonalization process and to guarantee identifiability. A confusion is made here by linking the existence of time-lag autocorrelation with the coherence time of a particular source signal, which is defined as a measure of characterization of the time varying nature of the channel due to the relative mobile velocity, and which is inversely proportional to the Doppler spread [54]. Nevertheless, the general idea here under the time-invariant assumption considered refers to the fact that large delay spreads are required in order to obtain sufficient cross-correlation for application of the said algorithms. Although such large delay spreads would indeed allow exploitation of larger lag autocorrelation evaluations, cross-correlation exists at a more fundamental level within the pulse-shaped nature of the received signals themselves and can be rightfully exploited even without delay spread (i.e. a coherent scenario).

Secondly, the authors also claim that communication signals sampled at the symbol rate are uncorrelated, and hence that higher lag autocorrelations cannot be exploited. This seemingly intuitive statement has its origins in the fact that the transmitted symbols $\left\{s_{g_{m}}\right\}_{m}$ of each source signal in (6) are uncorrelated, and therefore that cross-correlation evaluated at time lags greater or equal than $T$ is consequently nil. Such a reasoning is wrong considering that practical symbol pulse shaping waveforms temporally extend to much more than a symbol period, which is key to spectral efficiency (as explained in Section 2.2.2, see [54]). This implies that energy from a given symbol received at a time $t$ contributes to the source signal value at times up to $\pm W / 2$ (for symmetric pulses of width $W$ ), thus yielding cross-correlation for any general value of $W$. Moreover, it will be shown that autocorrelation of a source signal at lags equal to integer values of the symbol period (but within $\pm W$ ) is generally non-zero depending on the sampling phase of the considered signal.

Despite these odd claims however, the estimation principle of [18] based on AJD or on a PARAFAC analysis [55] from a set of zero-lag autocorrelation matrices remains nonetheless valid and practically functional. We will now proceed to the description and justification of the construction process of autocorrelation matrices to be considered in this paper and for subsequent processing in Section 4.

The received signal vector $x(t)$ in (4) is a cyclostationary random process of period $T$ with respect to $t$, whereas a sequence $\left\{x_{k}\right\}_{k=1}^{K}$ obtained by sampling of the same vector at a rate $1 / T_{S}=1 / T$ is a widesense stationary random process [41]. Such behaviour arise from the nature of $u_{g}(t)$ in (6), where pulses of iid amplitudes equally spaced in time implies that the signal statistical properties vary cyclically over time.

The cyclostationary nature of communication signals has been widely studied in the literature and exploited to various estimation purposes. A review of popular algorithms and techniques will not be presented in this section but the reader is referred to [56] and references therein for a comprehensive coverage on the subject.

In this paper we consider a general array autocorrelation matrix of the form:

$$
\begin{aligned}
\boldsymbol{R}_{x x}(t, \tau) & =E\left\{\boldsymbol{x}(t) \boldsymbol{x}^{\dagger}(t+\tau)\right\} \\
& =\boldsymbol{B} E\left\{\boldsymbol{u}(t) \boldsymbol{u}^{\dagger}(t+\tau)\right\} \boldsymbol{B}^{\dagger}+E\left\{\boldsymbol{n}(t) \boldsymbol{n}^{\dagger}(t+\tau)\right\} \\
& \equiv \boldsymbol{B} \boldsymbol{R}_{u u}(t, \tau) \boldsymbol{B}^{\dagger}+\boldsymbol{R}_{n n}(t, \tau)
\end{aligned}
$$

which depends on both $t$ and $\tau$. Note that since $\left\{u_{g}(t)\right\}_{g=1}^{G}$ are independent random processes, $\boldsymbol{R}_{u u}(t, \tau)$ is always a diagonal matrix. To give a more intuitive picture of how the cyclostationarity property of the received signals under general asynchronous conditions is to be exploited via eq. (16), consider the computation of zero-lag autocorrelation matrices $\boldsymbol{R}_{x x_{1}}, \boldsymbol{R}_{x x_{1}}^{\prime}$ and $\boldsymbol{R}_{x x_{2}}^{\prime}$ of Fig. 1. Taking $t=0$ as the time of the first sample in each cases, we have:

$$
\begin{aligned}
\boldsymbol{R}_{x x_{1}}=\boldsymbol{R}_{x x}(0,0) & =\boldsymbol{B} \boldsymbol{R}_{u u}(0,0) \boldsymbol{B}^{\dagger}, \\
\boldsymbol{R}_{x x_{1}}^{\prime}=\boldsymbol{R}_{x x}^{\prime}(0,0) & =\boldsymbol{B} \boldsymbol{R}_{u u}^{\prime}(0,0) \boldsymbol{B}^{\dagger}, \\
\boldsymbol{R}_{x x_{2}}^{\prime}=\boldsymbol{R}_{x x}^{\prime}(T / 2,0) & =\boldsymbol{B} \boldsymbol{R}_{u u}^{\prime}(T / 2,0) \boldsymbol{B}^{\dagger},
\end{aligned}
$$

since noise is neglected and since an upsampling factor $U=2$ is considered in (b). Under the synchronous conditions of case (a), sampling and symbol instants are coincident and consequently source signal values are restricted in $\{-1,1\}$ with equal probability. Therefore, $\operatorname{diag}\left\{\boldsymbol{R}_{u u}(0,0)\right\}=\left[\sigma_{\text {sym }_{1}}^{2} \sigma_{\text {sym }_{2}}^{2}\right]^{\dagger}$ where $\sigma_{\text {sym }}^{2}$ is the symbol power of the $g$-th source signal (in this case $\left.\sigma_{\text {sym }}^{2}=\sigma_{\text {sym }_{2}}^{2}=1\right)$. In (b), sampling and symbol instants of the sources are not coincident, and as a result $u_{g}\left(t_{k}\right) \notin\{-1,1\}^{6}$. We now have $\operatorname{diag}\left\{\boldsymbol{R}_{u u}^{\prime}(0,0)\right\}=$ $\left[\begin{array}{lll}a_{1_{1}} \sigma_{\text {sym }_{1}}^{2} & a_{1_{2}} \sigma_{\text {sym }}^{2}\end{array}\right]^{\top}$ where $1>a_{1_{1}} \approx 1$ since sampling instants are close to the symbol instants of $u_{1}(t)$, and where $a_{1_{2}}<a_{1_{1}}$. In the second sample sequence, $\operatorname{diag}\left\{\boldsymbol{R}_{u u}^{\prime}(T / 2,0)\right\}=\left[\begin{array}{lll}a_{21} \sigma_{\mathrm{sym}_{1}}^{2} & a_{2_{2}} \sigma_{\mathrm{sym}_{2}}^{2}\end{array}\right]^{\top}$ where this time $a_{21}$ has a substantially lower value than $a_{1_{1}}$ since the sample sequence is near $180^{\circ}$ out of phase with the symbol instants of $u_{1}(t)$ in a region where zerocrossings are most likely to occur. Consequently, it follows that signal $u_{1}(t)$ appears with a significantly lower power in $\boldsymbol{R}_{x x_{1}}^{\prime}$ than in $\boldsymbol{R}_{x x_{2}}^{\prime}$, whereas less importance changes are observed for $u_{2}(t)$. Although these observations are specific for the particular values of $\tau_{1}$ and $\tau_{2}$ considered in Fig. 1 (b), this analysis demonstrates that exploitation of sampling phase diversity is a simple and advantageous way of obtaining source power diverseness without requiring the need of real transmit power variations from the mobiles as exploited in [18]. Power diversity can also be obtained in the synchronous case of Fig. 1 (a), but matrices $\left\{\boldsymbol{R}_{u u}(t, 0)\right\} \forall t$ simply become scaled versions of each other if identically modulated signals are considered (i.e. if $p_{g}(t)=p(t) \forall g$ in (6)).

In this example, the effects of sampling phase diversity are best emphasized considering the zero-lag

${ }^{6}$ The exact pdf of a sample $u_{g}\left(t_{k}\right)$ could be obtained from $p_{g}(t)$ considering the set of consecutive symbols having a non-zero contribution to the source signal at time $t_{k}$. 
autocorrelation (power) of the source signals. However, the principle directly extends to any general value of $\tau$ in (16). To this point it is essential for a good understanding of the subsequent developments of the paper that one clearly distinguishes the separate effects of both $t$ and $\tau$ in $\boldsymbol{R}_{u u}(t, \tau)$ (or equivalently $\boldsymbol{R}_{x x}(t, \tau)$ ) from an intuitive point of view. Evaluation with respect to $t$ yields a periodic function in $T$ since signal values $\left\{u_{g}(t+k T)\right\}_{k \in \mathbb{Z}}$ in (6) are identically distributed considering a sufficiently large transmitted symbol sequence. On the other hand, evaluation of $\boldsymbol{R}_{u u}(t, \tau)$ with respect to $\tau$ represents the statistical correlation between $\boldsymbol{u}(t)$ and its delayed conjugate copy $\boldsymbol{u}^{*}(t+\tau)$. As $\tau$ increases (or decreases), $\boldsymbol{R}_{u u}(t, \tau)$ eventually goes to zero for any $t$ due to the finite width of $p_{g}(t) \forall g$.

As a means of better characterizing and comparing the statistical properties of the received source signals, we define a normalized autocorrelation profile (NAP) for each particular envelope $u_{g}(t)$ such that:

$$
\operatorname{NAP}_{g}(t, \tau) \triangleq \frac{E\left\{u_{g}(t) u_{g}(t+\tau)\right\}}{\sigma_{\text {sym }_{g}}^{2}}=\frac{\left[\boldsymbol{R}_{u u}(t, \tau)\right]_{g g}}{\sigma_{\text {sym }_{g}}^{2}}
$$

A complete theoretical derivation of (17) under the most general signal conditions is presented in Appendix, along with some discussions about important practical results. The NAP is a real function periodic in $t$ with period $T$, is independent of symbol power and provides insight as to how much autocorrelation diversity is to be obtained by sampling phase variation (when considered with respect to time). For example, Fig. 4 displays NAP evaluations for the most common pulseshaped signals (RC and Gaussian pulses) in a variety of conditions. It can be observed that the modulation parameters as well as the lengths and window types of pulse shaping functions have a marked effect on NAP diversity. Note that the normalization factor $1 / \sigma_{\text {sym }_{g}}^{2}$ in (17) could also be accounted for in $\boldsymbol{B}$ for each source signal as performed in [17]. We preferred however to adopt the actual notation to make a more intuitive distinction between channel and signal related properties.

The set of autocorrelation matrices to be considered for estimation of $\boldsymbol{B}$ via JD is directly obtained from (16) under general asynchronous signal conditions, where sampling phase variation is obtained by considering sets of distinct baud-sampled sequences from oversampling of $x(t)$ as explained in Section 2.2.2. Although exploitation of time lags greater than the symbol period is possible, as previously mentioned, we will nonetheless impose $\tau_{\max }=T$ for numerical computations in (16) considering that autocorrelation is likely to further decrease with larger lags, and also to limit the number of possible target matrices from which estimation of $\boldsymbol{B}$ is to be performed. Considering an integer oversampling factor $U$, a set of autocorrelation matrices is obtained as:

$$
\begin{aligned}
& \boldsymbol{R}_{x x}^{(p, q)} \triangleq \boldsymbol{R}_{x x}(t=p T / U, \tau=q T / U) \\
& \equiv \boldsymbol{B R}_{u u}^{(p, q)} \boldsymbol{B}^{\dagger}+\boldsymbol{R}_{n n}^{(p, q)}, \\
& p \in\{0,1, \ldots, U-1\}, q \in\{0,1, \ldots, U\},
\end{aligned}
$$

where time $t=0$ is taken as the first sampling instant. Fig. 5 depicts the general computation process in case

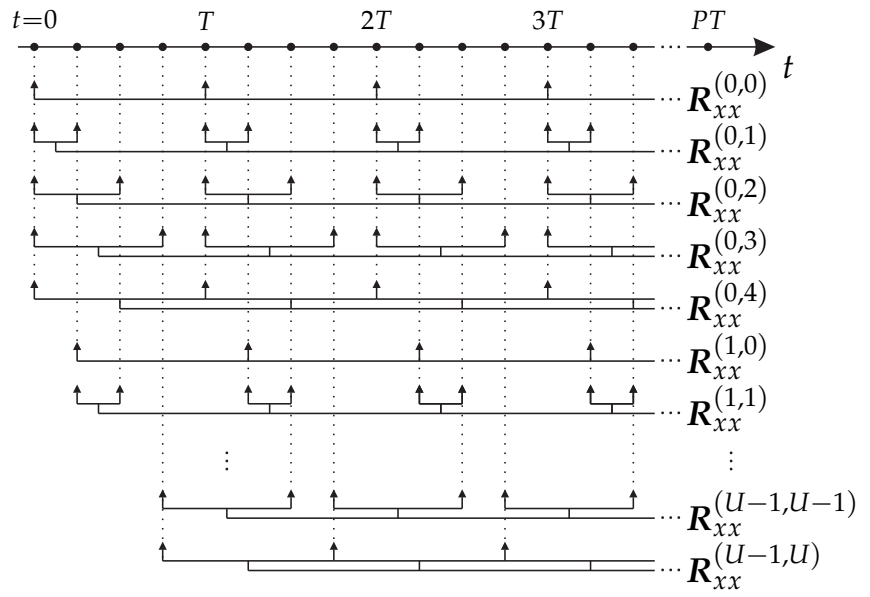

Figure 5. Construction principle of autocorrelation matrices (sampling instants) considering an upsampling factor $U=4$, a maximum time lag $\tau_{\max }=T$ and an observation time of $P$ symbol periods.

where $U=4$. In general, the combination of $U$ different sampling phases and $U+1$ distinct time lags allows the formation of $U(U+1)$ distinct autocorrelation matrices.

\section{Performing JDDTM}

\subsection{General Derivations}

Given (18), estimation of $\boldsymbol{B}$ can be performed from (18) by standard JD techniques similar to those considered in [18-21]. Alternatively, it could also be performed by a parallel factor analysis (PARAFAC) as considered in [18]. However, in either case one would first have to eliminate the noise term $\boldsymbol{R}_{n n}^{(p, q)}$ in (18) in order to obtain target matrices having the required eigenstructure. This process is carried on in the latter works by either assuming a temporally white noise ${ }^{7}$, implying that $\boldsymbol{R}_{n n}^{(p, q)}=\mathbf{0} \forall q \neq 0$, and/or performing noise variance estimation from an eigenvalue analysis of any $\boldsymbol{R}_{x x}^{(p, 0)}$ and then subtracting an estimate $\hat{\boldsymbol{R}}_{n n}^{(p, 0)}$ from $\boldsymbol{R}_{x x}^{(p, 0)} \forall p$. The applicability of this approach is severely limited by the noise distribution, and it also imposes additional constraints on the maximum number of source signals guaranteeing that at least one eigenvalue of $\boldsymbol{R}_{x x}^{(p, 0)}$ be associated with noise variance. In order to circumvent such limitations, we will adopt in this paper an estimation approach of $\boldsymbol{B}$ not directly drawn from (18), but rather based on a differential operation among appropriate matrices of the set. Recalling the stationary assumption on $n(t)$, it follows that:

$$
\boldsymbol{R}_{n n}^{\left(p_{1}, q\right)}=\boldsymbol{R}_{n n}^{\left(p_{2}, q\right)} \forall\left\{p_{1}, p_{2}\right\} \in\{0,1, \ldots, U-1\} ，
$$

since noise sample sequences are identically distributed $^{8}$ for any sampling phase index $p$. Therefore,

\footnotetext{
${ }^{7} \mathrm{~A}$ white noise has an infinite variance. The commonly encountered references to such types of noise is a language abuse to specify that a noise of finite variance is uncorrelated either temporally (between samples measured at different times) and/or spatially (between samples measured at different locations, e.g. on different elements).

${ }^{8}$ It is however sufficient for this property to be satisfied that $n(t)$ be cyclostationary with $t$ of period $T_{s}=T / U$ such that it remains identically distributed at each sampling instant.
} 
$\tau=0$
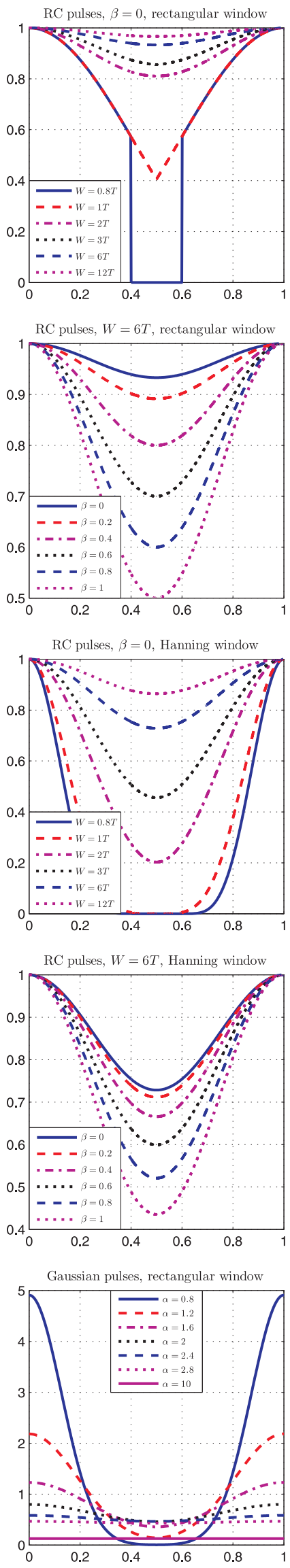

$\tau=T / 3$
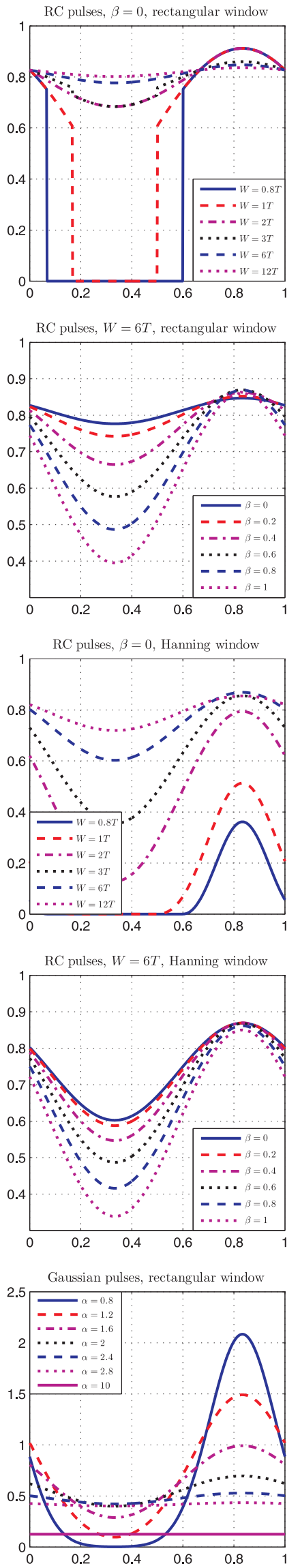

$\tau=2 T / 3$
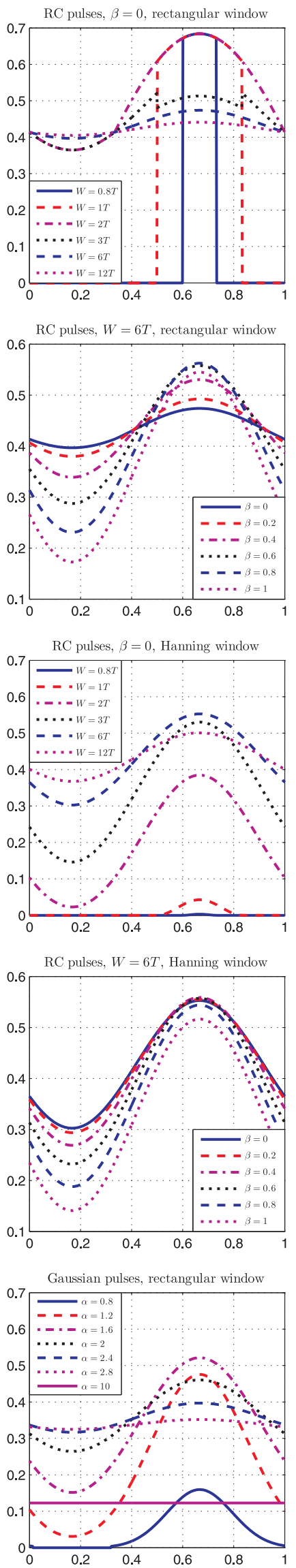

Figure 4. NAPs of differently modulated signals evaluated at time lags $\tau \in\{0, T / 3,2 T / 3\}$. Curves are plotted as a function of $t / T \in[0,1]$ where $t=0$ and $t=T$ correspond to symbol instants. For convenience, a symbol period of $T=1$ has been considered for Gaussian pulses. 
we can define a differential autocorrelation matrix:

$$
\begin{aligned}
\Delta \boldsymbol{R}_{x x}^{\left(p_{1}, p_{2}, q\right)} & \triangleq \boldsymbol{R}_{x x}^{\left(p_{1}, q\right)}-\boldsymbol{R}_{x x}^{\left(p_{2}, q\right)} \\
& =\boldsymbol{B}\left(\boldsymbol{R}_{u u}^{\left(p_{1}, q\right)}-\boldsymbol{R}_{u u}^{\left(p_{2}, q\right)}\right) \boldsymbol{B}^{\dagger}+\overbrace{\boldsymbol{R}_{n n}^{\left(p_{1}, q\right)}-\boldsymbol{R}_{n n}^{\left(p_{2}, q\right)}}^{\mathbf{0}} \\
& \equiv \boldsymbol{B} \Delta \boldsymbol{R}_{u u}^{\left(p_{1}, p_{2}, q\right)} \boldsymbol{B}^{\dagger}
\end{aligned}
$$

which now has a closer form to the ideal eigenstructure. The value of $\Delta \boldsymbol{R}_{u u}^{\left(p_{1}, p_{2}, q\right)}$ depends on the statistical nature of the source signals as well as asynchronous delays $\left\{\tau_{g}\right\}_{g=1}^{G}$, and is generally non-zero for $p_{1} \neq p_{2}$. The advantages of adopting a differential approach of the kind are:

1) Elimination of the need for noise variance estimation (assuming a spatially white noise of the type $\sigma_{n}^{2} \boldsymbol{I}$, see $\left.[17,18]\right)$. This operation is required when a zero-lag autocorrelation matrix is included in the set of target matrices.

2) Adaptability to virtually all types of noise distributions regardless of spatial or temporal correlation.

3) Exploitation of the complete degree of freedom of the array. Since the condition $G<N$ ensuring that the lowest eigenvalue of $\boldsymbol{R}_{x x}^{(p, 0)} \forall p$ be equal to the noise variance is no longer needed, up to $G=N$ source signals can be considered.

Note that eq. (20) cannot be exploited under assumption of a stationary observable vector $x(t)$ (considering possible application of the algorithm in contexts other than digital communications), since it would imply that $\boldsymbol{R}_{u u}^{\left(p_{1}, q\right)}=\boldsymbol{R}_{u u}^{\left(p_{2}, q\right)} \forall\left\{p_{1}, p_{2}\right\}$ and consequently that $\Delta \boldsymbol{R}_{x x}^{\left(p_{1}, p_{2}, q\right)}=\mathbf{0}$ in (20). The practicability of our approach relies on natural autocorrelation diversity provided by the cyclostationary nature of communication signals in a general asynchronous context where $\Delta \boldsymbol{R}_{x x}^{\left(p_{1}, p_{2}, q\right)}$ is generally non-zero.

An examination of eq. (20) shows that $\Delta \boldsymbol{R}_{x x}^{\left(p_{1}, p_{2}, q\right)}=$ $-\Delta \boldsymbol{R}_{x x}^{\left(p_{2}, p_{1}, q\right)}$. Hence either one of such matrices is of potential use for estimation of $\boldsymbol{B}$. In general, among the set of $U$ autocorrelation matrices obtained at different sampling phases $p$ for each time lag $q, U^{2}$ differential autocorrelation matrices $\Delta \boldsymbol{R}_{x x}^{\left(p_{1}, p_{2}, q\right)}$ can be obtained from (20) but only $U(U-1) / 2$ effective ones will be retained for the JD process:

$$
\left\{\Delta \boldsymbol{R}_{x x}^{\left(p_{1}, p_{2}, q\right)}\right\} \begin{aligned}
& p_{1} \in\{0,1, \ldots, U-2\} \\
& p_{1}<p_{2} \leqslant U-1
\end{aligned} .
$$

Considering the $U+1$ distinct time lag values in (18), the maximum number of DTM $D$ to be considered for estimation of $\boldsymbol{B}$ is hence given by:

$$
D=\frac{1}{2} U(U-1)(U+1) .
$$

Table I lists some evaluations of (22), and exemplifies the rapid increase of $D$ with $U$. It also follows from (22) that an upsampling factor $U \geqslant 2$ is necessary for application of the algorithm.

The literature provides a wide spectrum of AJD techniques with a particular emphasis on the BSS problem [22-30] (more particularly, see [22] for a short summary of popular existing algorithms on the subject).
Table I

UpSAMPLING FACtor $U$ ANd CORRESPONdING MAXimum Number of DTM D

\begin{tabular}{c||c|c|c|c|c}
$U$ & 2 & 3 & 4 & 5 & 6 \\
\hline$D$ & 3 & 12 & 30 & 60 & 105
\end{tabular}

Given a set $\left\{\boldsymbol{R}_{d}=\mathbf{Q} \boldsymbol{\Lambda}_{d} \mathbf{Q}^{\dagger}\right\}_{d=1}^{D}$ of target matrices such that $Q \in \mathbb{C}^{N \times N}$ and $\Lambda_{d} \forall d$ is diagonal, the JD process consists in determining a joint diagonalizer $V$ such that $\boldsymbol{V} \boldsymbol{R}_{d} \boldsymbol{V}^{\dagger} \forall d$ is also a diagonal matrix. An ideal solution has the form $V=Z Q^{-1}$ where $Z$ is a scale permutation matrix containing only one non-zero element per line and column. An estimation of $Q$ is then obtained as $\hat{Q}=V^{-1}$.

When the set $\left\{\boldsymbol{R}_{d}\right\}_{d=1}^{D}$ is not composed of ideal eigenmatrices (e.g. the case of autocorrelation matrix estimates obtained by a finite number of samples), the operation is rather referred to as AJD. The differences between existing AJD algorithms essentially lie in the choice or implementation of an appropriate optimization criterion, the most popular being the minimization of the off-diagonal element amplitudes of the set $\left\{\boldsymbol{V} \boldsymbol{R}_{d} \boldsymbol{V}^{\dagger}\right\}_{d=1}^{D}[22,25]$.

For convenience, most AJD algorithms assume that the mixing or channel matrix is full-rank and invertible. This is not the case for $\boldsymbol{B}$ in (21) since $\operatorname{rank}\{\boldsymbol{B}\}=\min (N, G)$. The set of matrices $\Delta \boldsymbol{R}_{x x}^{\left(p_{1}, p_{2}, q\right)}$ can be adapted to such a compatible form by noting that the columns of $\boldsymbol{B}$ lies in the signal subspace. More specifically, expressing a given $\Delta \boldsymbol{R}_{x x}^{\left(p_{1}, p_{2}, q\right)}$ in (21) in terms of its eigenvalue decomposition (EVD) yields:

$$
\begin{aligned}
\Delta \boldsymbol{R}_{x x}^{\left(p_{1}, p_{2}, q\right)}= & {\left[\begin{array}{ll}
\boldsymbol{E}_{s}^{\left(p_{1}, p_{2}, q\right)} & \boldsymbol{E}_{n}^{\left(p_{1}, p_{2}, q\right)}
\end{array}\right] . } \\
& {\left[\begin{array}{cc}
\boldsymbol{\Lambda}_{s}^{\left(p_{1}, p_{2}, q\right)} & \mathbf{0} \\
\mathbf{0} & \mathbf{0}
\end{array}\right]\left[\begin{array}{l}
\left(\boldsymbol{E}_{s}^{\left(p_{1}, p_{2}, q\right)}\right)^{\dagger} \\
\left(\boldsymbol{E}_{n}^{\left(p_{1}, p_{2}, q\right)}\right)^{\dagger}
\end{array}\right] } \\
= & \boldsymbol{E}_{s}^{\left(p_{1}, p_{2}, q\right)} \boldsymbol{\Lambda}_{s}^{\left(p_{1}, p_{2}, q\right)}\left(\boldsymbol{E}_{s}^{\left(p_{1}, p_{2}, q\right)}\right)^{\dagger},
\end{aligned}
$$

where $\boldsymbol{E}_{s}^{\left(p_{1}, p_{2}, q\right)} \in \mathbb{C}^{N \times G}$ represents a set of orthonormal basis vectors of the signal subspace ${ }^{9}$. From (20) and (23), it follows that $\boldsymbol{B}$ can be expressed in terms of $\boldsymbol{E}_{S}^{\left(p_{1}, p_{2}, q\right)}$ such that:

$$
\boldsymbol{B}=\boldsymbol{E}_{S}^{\left(p_{1}, p_{2}, q\right)} \boldsymbol{Q}^{\left(p_{1}, p_{2}, q\right)},
$$

where $Q^{\left(p_{1}, p_{2}, q\right)}$ is a $G \times G$ coefficient matrix. Alternatively, a set of orthonormal basis vectors of the signal subspace may also be obtained not from a single, but from the joint use of all $D$ matrices $\Delta \boldsymbol{R}_{x x}^{\left(p_{1}, p_{2}, q\right)}$ in (21) (considering all $q \in\{0,1, \ldots, U\}$ ). Such an approach is furthermore desirable considering the use of practical matrix estimates obtained by a finite number of samples where the choice of an optimal target matrix $\Delta \boldsymbol{R}_{x x}^{\left(p_{1}, p_{2}, q\right)}$ in (23) could be ambiguous. To this end, one can express

${ }^{9}$ The dimension of the signal subspace estimated from $\Delta \boldsymbol{R}_{x x}^{\left(p_{1}, p_{2}, q\right)}$ in (21) is generally $G$ under natural asynchronous conditions, but a finite set of specific values of $\left\{\tau_{g}\right\}_{g=1}^{G}$ for identically modulated signals can reduce this number. These aspects are discussed in more details in Section 4.3. 
the joint singular value decomposition (SVD) of the line-wise concatenation of all $D$ target matrices such that:

$$
\begin{aligned}
\boldsymbol{U} \boldsymbol{S} \boldsymbol{V}^{\dagger} & =\operatorname{SVD}\left[\left\{\Delta \boldsymbol{R}_{x x}^{\left(p_{1}, p_{2}, q\right)}\right\} \begin{array}{l}
q \in\{0,1, \ldots, U\} \\
p_{1} \in\{0,1, \ldots, U-2\} \\
p_{1}<p_{2} \leqslant U-1
\end{array}\right] \\
& =\left[\begin{array}{ll}
\boldsymbol{U}_{S} & \boldsymbol{U}_{n}
\end{array}\right]\left[\begin{array}{cc}
\boldsymbol{S}_{S} & \mathbf{0} \\
\mathbf{0} & \mathbf{0}
\end{array}\right]\left[\begin{array}{c}
\boldsymbol{V}_{S}^{\dagger} \\
\boldsymbol{V}_{n}^{\dagger}
\end{array}\right] \\
& =\boldsymbol{U}_{S} \boldsymbol{S}_{S} \boldsymbol{V}_{S}^{\dagger},
\end{aligned}
$$

where $\boldsymbol{U}_{s} \in \mathbb{C}^{N \times G}$ as in (23) since $\boldsymbol{S}_{s}$ is generally $G \times G$. A more representative set of orthonormal basis vectors of the signal subspace may hence be taken as:

$$
E_{s}=U_{s}
$$

such that:

$$
B=E_{s} Q \Rightarrow E_{S}^{\dagger} B=Q
$$

The set of target matrices to be considered for JD is then be obtained as:

$$
S_{D} \triangleq\left\{\boldsymbol{E}_{s}^{\dagger} \Delta \boldsymbol{R}_{x x}^{(d)} \boldsymbol{E}_{s}=\boldsymbol{Q} \Delta \boldsymbol{R}_{u u}^{(d)} \boldsymbol{Q}^{\dagger}\right\}_{d=1}^{D},
$$

where

$$
\begin{aligned}
& \left\{\Delta \boldsymbol{R}_{x x}^{(d)}, \Delta \boldsymbol{R}_{u u}^{(d)}\right\}_{d=1}^{D}= \\
& \left\{\Delta \boldsymbol{R}_{x x}^{\left(p_{1}, p_{2}, q\right)}, \Delta \boldsymbol{R}_{u u}^{\left(p_{1}, p_{2}, q\right)}\right\} \begin{array}{l}
q \in\{0,1, \ldots, U\} \\
p_{1} \in\{0,1, \ldots, U-2\} \\
p_{1}<p_{2} \leqslant U-1
\end{array}
\end{aligned}
$$

The BSS problem hence reduces to the estimation of $Q \in \mathbb{C}^{G \times G}$ (and subsequently $\boldsymbol{B}$ in (27)), which is nonsingular if $\boldsymbol{B}$ has full column rank. Note that this operation also reduces the computational complexity of the AJD operation given the generally reduced dimensions of $\boldsymbol{Q}_{s} \Delta \boldsymbol{R}_{u u}^{\left(p_{1}, p_{2}, q\right)} \boldsymbol{Q}_{s}^{\dagger}$ in (28). In essence, the procedure resembles the whitening process considered in [17] without however requiring $Q$ to be unitary (only full-rank), a property that has been identified to restrict the performance of related BSS algorithms [57]. Upon obtaining an estimate $\hat{Q}$ of $Q$ by AJD of (28), an estimate $\hat{\boldsymbol{B}}$ of $\boldsymbol{B}$ is obtained as:

$$
\hat{B}=E_{S} \hat{Q} \text {. }
$$

\subsection{A Set of "More Representative" Target Matrices}

Consider application of the algorithm with a very large oversampling factor $U$. The time $T_{S}=T / U$ separating two samples is consequently very small, and as a result matrices $\boldsymbol{R}_{u u}^{(p, q)}$ and $\boldsymbol{R}_{u u}^{(p+1, q)}$ for $p \leqslant$ $U-2$ in (18) take very similar values, implying that $\boldsymbol{R}_{x x}^{(p, q)} \approx \boldsymbol{R}_{x x}^{(p+1, q)}$ (refer to Figs. 1 (b) and 5). One therefore obtains $\Delta \boldsymbol{R}_{x x}^{(p, p+1, q)} \approx \mathbf{0}$ in (20) and likewise $Q_{s} \Delta \boldsymbol{R}_{u u}^{(p, p+1, q)} \boldsymbol{Q}_{s}^{\dagger} \approx \mathbf{0}$ in (29). The set of target matrices to be considered for AJD thus encompasses a mixture of arbitrarily low-valued element matrices (of low relevance), as well as better conditioned matrices resulting from higher statistical diversity obtained at different sampling phases. A similar situation could also arise for a low value of $U$ if appropriate conditions on $\left\{\tau_{g}\right\}_{g=1}^{G}$ are satisfied for particular values of $p_{1}$ and $p_{2}$ in (21).
The work of Yeredor in [58] proves particularly useful in such a context since it allows the computation of two "more representative" matrices from the set of initial target matrices, which can then be considered for AJD. The procedure is shown to be optimal in the least-squares (LS) sense for target matrices of size of $2 \times 2$, and to yield fairly "good" results in larger scale problems such as herein considered (in general). Given its potential utility in (28), a brief description of the main guidelines of this procedure will now be presented.

The principle is inspired from [59], and based on the minimization of a direct least-squares (DLS) criterion defined as:

$$
\mathcal{C}_{\mathrm{DLS}}\left(\hat{\boldsymbol{Q}},\left\{\Delta \hat{\boldsymbol{R}}_{u u}^{(d)}\right\}_{d=1}^{D}\right) \triangleq \sum_{d=1}^{D}\left\|\Delta \boldsymbol{R}_{x x}^{(d)}-\hat{\boldsymbol{Q}} \Delta \hat{\boldsymbol{R}}_{u u}^{(d)} \hat{\boldsymbol{Q}}^{\dagger}\right\|_{F}^{2},
$$

where $\|\cdot\|_{F}$ denotes the Frobenius norm. Using the vector operator $\operatorname{vec}(\cdot): \mathbb{C}^{N \times M} \rightarrow \mathbb{C}^{N M \times 1}$ and the Khatri-Rao product of two matrices (in this case defined as a matrix $\boldsymbol{A} \circ \boldsymbol{B}=\left[\boldsymbol{a}_{1} \otimes \boldsymbol{b}_{1}, \boldsymbol{a}_{2} \otimes \boldsymbol{b}_{2}, \ldots\right]$ where ' $\otimes$ ' denotes the Kronecker product), it is shown that each term of the $\mathcal{C}_{\text {DLS }}$ sum can be equivalently expressed as:

$$
\left\|\Delta \boldsymbol{R}_{x x}^{(d)}-\hat{\mathbf{Q}} \Delta \hat{\boldsymbol{R}}_{u u}^{(d)} \hat{\boldsymbol{Q}}^{\dagger}\right\|_{F}^{2}=\left\|\boldsymbol{m}_{d}-\hat{\mathcal{Q}} \hat{\boldsymbol{p}}_{d}\right\|_{F}^{2},
$$

where $\boldsymbol{m}_{d}=\operatorname{vec}\left\{\Delta \boldsymbol{R}_{x x}^{(d)}\right\}, \hat{\mathcal{Q}}=\hat{\boldsymbol{Q}}^{*} \circ \hat{\boldsymbol{Q}}$, and $\hat{\boldsymbol{p}}_{d}=$ $\operatorname{diag}\left\{\Delta \hat{\boldsymbol{R}}_{u u}^{(d)}\right\}$. The parameters $\left\{\Delta \hat{\boldsymbol{R}}_{u u}^{(d)}\right\}_{d=1}^{D}$ are generally not of interest in a BSS problem, and consequently minimization of (31) can be performed for each term of the sum with respect to $\Delta \hat{\boldsymbol{R}}_{u u}^{(d)}$ (or $\hat{\boldsymbol{p}}_{d}$ in (32)). We have:

$$
\boldsymbol{m}_{d}-\hat{\mathcal{Q}} \hat{\boldsymbol{p}}_{d}=0 \Rightarrow \hat{\boldsymbol{p}}_{d} \approx\left(\hat{\mathcal{Q}}^{\dagger} \hat{\mathcal{Q}}\right)^{-1} \hat{\mathcal{Q}}^{\dagger} \boldsymbol{m}_{d}=\hat{\mathcal{Q}}^{\#} \boldsymbol{m}_{d}
$$

which is an optimal solution in the LS sense. Substituting (33) and (32) into (31), we get:

$$
\mathcal{C}_{\mathrm{DLS}}(\hat{\boldsymbol{Q}})=\sum_{d=1}^{D}\left\|\boldsymbol{m}_{d}-\hat{\mathcal{Q}} \hat{\mathcal{Q}}^{\#} \boldsymbol{m}_{d}\right\|_{F}^{2}=\sum_{d=1}^{D}\left\|\boldsymbol{P}^{\perp}(\hat{\mathcal{Q}}) \boldsymbol{m}_{d}\right\|_{F}^{2},
$$

where $\boldsymbol{P}^{\perp}(\hat{\mathcal{Q}})=\boldsymbol{I}-\hat{\mathcal{Q}} \hat{\mathcal{Q}}^{\#}$ is Hermitian and idempotent (i.e. $\left.\left(\boldsymbol{P}^{\perp}(\hat{\mathcal{Q}})\right)^{2}=\boldsymbol{P}^{\perp}(\hat{\mathcal{Q}})\right)$. As a result, it follows that:

$$
\begin{aligned}
\mathcal{C}_{\mathrm{DLS}}(\hat{\boldsymbol{Q}}) & =\sum_{d=1}^{D}\left\|\boldsymbol{m}_{d}^{\dagger} \boldsymbol{P}^{\perp}(\hat{\mathcal{Q}}) \boldsymbol{m}_{d}\right\|_{F} \\
& =\operatorname{trace}\left\{\boldsymbol{P}^{\perp}(\hat{\mathcal{Q}}) \sum_{d=1}^{D} \boldsymbol{m}_{d} \boldsymbol{m}_{d}^{\dagger}\right\} \\
& \equiv \operatorname{trace}\left\{\boldsymbol{P}^{\perp}(\hat{\mathcal{Q}}) \boldsymbol{Y}\right\} .
\end{aligned}
$$

The EVD of $\boldsymbol{Y}$ in (35) can be expressed as:

$$
\begin{aligned}
& \boldsymbol{Y}=\boldsymbol{V} \boldsymbol{\Lambda} \boldsymbol{V}^{\dagger}=\sum_{m=1}^{G^{2}} \lambda_{m} \boldsymbol{v}_{m} \boldsymbol{v}_{m}^{\dagger}, \\
& \lambda_{1} \geqslant \lambda_{2} \geqslant \ldots, \geqslant \lambda_{G^{2}} \geqslant 0,
\end{aligned}
$$

where it is assumed that $\hat{Q} \in \mathbb{C}^{G \times G}(\hat{G}=G)$ such that $m_{d} \in \mathbb{C}^{G^{2} \times 1}$. Yeredor then shows that a lower bound on 
$\mathcal{C}_{\text {DLS }}$ is given by:

$$
\mathcal{C}_{\text {DLS }}(\hat{\mathbf{Q}}) \geqslant \sum_{m=G+1}^{G^{2}} \lambda_{m}
$$

The result follows from noting that $\operatorname{rank}\{\hat{\mathcal{Q}}\}=$ $\operatorname{rank}\{\hat{\boldsymbol{Q}}\}$, and consequently that $\operatorname{rank}\left\{\boldsymbol{P}^{\perp}(\hat{\mathcal{Q}})\right\} \geqslant G^{2}-$ $\operatorname{rank}\{\hat{Q}\} \equiv r$. Recalling that the eigenvalues of an idempotent matrix are either 0 or 1 , it follows that $P^{\perp}(\hat{\mathcal{Q}})$ can be expressed by its EVD such that $\boldsymbol{P}^{\perp}(\hat{\mathcal{Q}})=\boldsymbol{S}(\hat{\mathcal{Q}}) S^{\dagger}(\hat{\mathcal{Q}})$, where $\boldsymbol{S}(\hat{\mathcal{Q}}) \in \mathbb{C}^{G^{2} \times r}$ satisfies $S^{\dagger}(\hat{\mathcal{Q}}) S(\hat{\mathcal{Q}})=I$. Combining those results and (36) in (35) yields:

$$
\begin{aligned}
\mathcal{C}_{\mathrm{DLS}}(\hat{\boldsymbol{Q}}) & =\operatorname{trace}\left\{\boldsymbol{W}(\hat{\mathcal{Q}}) \boldsymbol{\Lambda} \boldsymbol{W}^{\dagger}(\hat{\mathcal{Q}})\right\} \\
& =\sum_{m=1}^{\mathrm{G}^{2}} \lambda_{m} \boldsymbol{w}_{m}^{\dagger}(\hat{\mathcal{Q}}) \boldsymbol{w}_{m}(\hat{\mathcal{Q}}),
\end{aligned}
$$

where $\boldsymbol{W}(\hat{\mathcal{Q}})=\boldsymbol{S}^{\dagger}(\hat{\mathcal{Q}}) \boldsymbol{V} \in \mathbb{C}^{r \times G^{2}}$ and where $\boldsymbol{w}_{m}(\hat{\mathcal{Q}})$ is the $m$-th column of $\boldsymbol{W}(\hat{\mathcal{Q}})$, satisfying $\left\|\boldsymbol{w}_{m}(\hat{\mathcal{Q}})\right\| \leqslant 1$. Given $\|\boldsymbol{W}(\hat{\mathcal{Q}})\|_{F}^{2}=r$, a minimum value of $\mathcal{C}_{\mathrm{DLS}}(\hat{\boldsymbol{Q}})$ is obtained when the weights $\boldsymbol{w}_{m}^{\dagger}(\hat{\mathcal{Q}}) \boldsymbol{w}_{m}(\hat{\mathcal{Q}})$ of the last $r \geqslant G^{2}-G$ eigenvalues $\lambda_{m}$ in (38) are equal to 1 (and the others zero), thus yielding (37). Further derivations in [58] show that this bound can almost always be reached for target matrices $\left\{\boldsymbol{E}_{s}^{\dagger} \Delta \boldsymbol{R}_{x x}^{(d)} \boldsymbol{E}_{s}\right\}_{d=1}^{D}$ of size $2 \times 2$ for any $D$ if $\hat{Q}$ is taken as the solution of the exact JD of matrices

$$
S_{2} \triangleq\left\{\operatorname{unvec}\left\{\boldsymbol{v}_{1}\right\}, \text { unvec }\left\{\boldsymbol{v}_{2}\right\}\right\}
$$

from (36), where the inverse vector operator satisfies unvec $\{\operatorname{vec}\{\boldsymbol{X}\}\}=\boldsymbol{X}$. For larger target matrices $(G>2)$, an estimate $\hat{Q}$ likewise obtained is shown to be "pretty good", or can serve as an initial guess for a subsequent iterative AJD algorithm.

Estimation performance considering AJD of the full and the "more representative" sets of target matrices $\left(S_{D}\right.$ in (28) and $S_{2}$ in (39)) will be compared in more details in Section 5.

\subsection{Identifiability}

The identifiability of $\boldsymbol{B}$ to within column scale and permutation is guaranteed provided that the set of target matrices $\left\{\boldsymbol{Q} \Delta \boldsymbol{R}_{u u}^{(d)} \boldsymbol{Q}^{\dagger}\right\}_{d=1}^{D}$ in (28) be jointly diagonalizable. In such a case, an estimate $\hat{Q}$ of $Q$ possesses the form:

$$
\hat{Q}=Q Z,
$$

where $\mathbf{Z}$ is a scale permutation matrix containing only one non-zero element per line and column (see Section 4.1). $\hat{Q}$ is then said to be essentially equal to $Q$ [17], and as a result we also have $\hat{\boldsymbol{B}}=\boldsymbol{B Z}$ in (30). This section is intended at discussing the necessary conditions to guarantee identifiability of $\boldsymbol{B}$ given the general signal model of eqs. (4) and (6).

A first requirement is that $B$ be full column rank such that $Q$ be invertible in (27), and thus suitable for a JD process. The nonsingularity of the mixing (or channel) matrix is an assumption made in a majority of BSS algorithms, and brings no particular limitation given the continuous and somewhat arbitrary nature of coefficients $[B]_{p q}$ that would arise in a practical situation.

Given $\operatorname{rank}\{\boldsymbol{Q}\}=G$, the study of identifiability conditions can be restricted to the particular structure of $\left\{\Delta \boldsymbol{R}_{u u}^{(d)} Q\right\}_{d=1}^{D}$ in (28). It is of course necessary that the latter matrices be diagonal, which is ensured by the uncorrelated assumption of the source signals, i.e. $E\left\{u_{p}(t) u_{q}(t)\right\}=\sigma_{u_{p}}^{2}(t) \delta_{p, q}$. Note also that the diagonal elements of $\boldsymbol{R}_{u u}(t, \tau)$ in (16) are real valued (see eq. (A.4) in Appendix), implying that $\Delta \boldsymbol{R}_{u u}^{(d)} \in$ $\mathbb{R}^{G \times G} \forall d$. Some general requirements on the source signals for identifiability of the mixing matrix in JDbased BSS algorithms are discussed in [22]. However, [17] gives a more rigorous criterion in this regard in the particular case where $Q$ is unitary. Although this condition is not generally satisfied in (27), we will nontheless present a review of this criterion which will subsequently prove helpful in deriving the necessary identifiability conditions in the general case of eq. (28). It is shown in [17], eq. (21), that identifiability of a unitary $Q$ to within column scale and permutation is possible if and only if

$$
\exists d \mid\left[\Delta \boldsymbol{R}_{u u}^{(d)}\right]_{p p} \neq\left[\Delta \boldsymbol{R}_{u u}^{(d)}\right]_{q q} \forall p \neq q,
$$

i.e. if there exists at least one matrix $\Delta \boldsymbol{R}_{u u}^{(d)}, d \in$ $\{1,2, \ldots, D\}$, having distinct diagonal elements. It can be shown however that this condition does not always yield accurate identifiability predictions in the most general cases, as it is sometimes too restrictive in regard of the structure of $\left\{\Delta \boldsymbol{R}_{u u}^{(d)}\right\}_{d=1}^{D}$. For example, the set of target matrices

$$
\left\{Q\left[\begin{array}{lll}
1 & 0 & 0 \\
0 & 0 & 0 \\
0 & 0 & 0
\end{array}\right] Q^{\dagger}, Q\left[\begin{array}{lll}
0 & 0 & 0 \\
0 & 1 & 0 \\
0 & 0 & 0
\end{array}\right] Q^{\dagger}, Q\left[\begin{array}{lll}
0 & 0 & 0 \\
0 & 0 & 0 \\
0 & 0 & 1
\end{array}\right] Q^{\dagger}\right\}
$$

is jointly diagonalizable in the sense of essential equality for any full-rank $Q \in \mathbb{C}^{3 \times 3}$ (unitary or not) even if none of the inner diagonal matrices satisfies (41). Otherwise, as shown in Appendix B of [17], eq. (41) correctly predicts a problem solvability for any unitary $Q$. In the context of this paper however, eq. (41) cannot apply since $Q$ is generally not unitary, but it can nonetheless serve as a good starting point to derive a somewhat similar identifiability criterion for the set of DTM (28).

Consider in this vein any full-rank and non-unitary $Q$, and suppose that $\left[\Delta \boldsymbol{R}_{u u}^{(d)}\right]_{11}=0 \forall d$. Even if a given $\Delta \boldsymbol{R}_{u u}^{(d)}$ satisfies (41), there exists no possibility of identifying $q_{1}$ (the first column of $Q$ ) to within an arbitrary non-zero scale factor since no information about this component is present in the set of target matrices. However, if $Q$ was unitary, identifiability could still be possible considering the property of orthogonality between distinct lines and columns of a unitary matrix. Another example would be that of a set of target matrices of the form $\left\{Q D Q^{\dagger}, Q(\alpha D) Q^{\dagger}\right\}$, where $D$ satisfies (41). Identifiability of $Q$ cannot be performed either unless the columns of $Q$ are orthogonal, in which 
case the eigenvector matrix of either (if $\alpha \neq 0$ ) target matrices can be taken as $\hat{Q}$ and be essentially equal to $Q$.

From the latter considerations and accounting for the presence of arbitrary numbers of zeros in $\operatorname{diag}\left\{\Delta \boldsymbol{R}_{u u}^{(d)}\right\} \forall d$ given the differential approach of eq. (20), a general identifiability criterion may now be formulated inspired from [17] for any full-rank $Q \in \mathbb{C}^{G \times G}$ and diagonal $\Delta \boldsymbol{R}_{u u}^{(d)} \in \mathbb{R}^{G \times G}$ in (28). First let the $d$-th row of a matrix $M \in \mathbb{R}^{D \times G}$ correspond to the diagonal elements of the $d$-th target matrix:

$$
\begin{aligned}
& {\left[\begin{array}{llll}
M_{d 1} & M_{d 2} & \ldots & M_{d G}
\end{array}\right]=\operatorname{diag}\left\{\Delta \boldsymbol{R}_{u u}^{(d)}\right\}^{\top},} \\
& d \in\{1,2, \ldots, D\} \text {. }
\end{aligned}
$$

Then a non-zero joint diagonalizer of the form $\hat{Q}^{-1}=$ $(Q Z)^{-1}$ exists for the set of target matrices (28) if and only if

$$
\boldsymbol{M}=\left[\begin{array}{cc|c|cc}
M_{11} & \ldots & M_{1 g} & \ldots & M_{1 G} \\
\vdots & & \vdots & & \vdots \\
\hline M_{d 1} & \ldots & M_{d g} & \ldots & M_{d G} \\
\hline \vdots & & \vdots & & \vdots \\
M_{D 1} & \ldots & \frac{M_{D g}}{\hat{m}_{g}} & \ldots & M_{D G}
\end{array}\right]<\operatorname{diag}\left\{\Delta \boldsymbol{R}_{u u}^{(d)}\right\}^{\top}
$$

Figure 6. Representation of matrix $\boldsymbol{M}$ with corresponding $g$-th column and $d$-th line.

$$
\operatorname{rank}\left\{\left[\begin{array}{ll}
\boldsymbol{m}_{g_{1}} & \boldsymbol{m}_{g_{2}}
\end{array}\right]\right\}=2 \forall 1 \leqslant g_{1} \neq g_{2} \leqslant G,
$$

where $m_{g}$ is the $g$-th column of $M$ (refer to Fig. 6 for a structural representation). Note that this general compact-form identifiability condition does not impose a given $\Delta \boldsymbol{R}_{u u}^{(d)}$ to possess distinct diagonal elements as in (41), and also allows that a number of matrices $\Delta \boldsymbol{R}_{u u}^{(d)}=\mathbf{0} \forall d \in\left\{1,2, \ldots, d_{c}\right\}$ provided that a matrix $\boldsymbol{M}^{\prime}$ constructed from $\Delta \boldsymbol{R}_{u \mathfrak{u}}^{(d)} \forall d \in\left\{d_{c}+1, d_{c}+2, \ldots, D\right\}$ satisfies (44). In the example of eq. (42), we have:

$$
\boldsymbol{M}=\left[\begin{array}{lll}
1 & 0 & 0 \\
0 & 1 & 0 \\
0 & 0 & 1
\end{array}\right]=\boldsymbol{I},
$$

which is full-rank and satisfies (44). An identical result would also be obtained if any number of zero matrices would be added in (42).

One also deduces from (44) that identifiability cannot be achieved if each component of $Q$ is not adequately represented in (28), e.g. if $\boldsymbol{m}_{g}=\mathbf{0}$ for any $g$, or more generally if $\boldsymbol{m}_{g_{1}}=\alpha \boldsymbol{m}_{g_{2}} \forall\left\{g_{1}, g_{2}\right\}$. Interestingly, we note that for $D \geqslant G$, eq. (44) reduces to $\operatorname{rank}\{\boldsymbol{M}\}=G$.

Although (44) gives the necessary and sufficient requirement on $\left\{\Delta \boldsymbol{R}_{u u}^{(d)}\right\}_{d=1}^{D}$ to guarantee identifiability (given $\operatorname{rank}\{\boldsymbol{Q}\}=G$ ), no general condition has yet been formulated with respect to the source model of eq. (6), which solely determines the structure of $\boldsymbol{R}_{u u}(t, \tau)$ in (16). Suppose in this regard that the sources are synchronous (considering any sampling phase or oversampling factor) and have identical pulse shaping functions, i.e. $\tau_{g}=\tau_{0}$ and $\left.p_{g}(t)=p(t)\right\} \forall g$. Hence $\operatorname{NAP}_{g}(t, \tau)=\operatorname{NAP}(t, \tau) \forall g$ and it follows from (17) that

$$
\left[\boldsymbol{R}_{u u}(t, \tau)\right]_{g g}=\sigma_{\text {sym }_{g}}^{2} \operatorname{NAP}(t, \tau),
$$

and consequently

$$
\operatorname{diag}\left\{\boldsymbol{R}_{u u}(t, \tau)\right\}=\operatorname{NAP}(t, \tau)\left[\begin{array}{llll}
\sigma_{\text {sym }_{1}}^{2} & \sigma_{\text {sym }_{2}}^{2} & \ldots & \sigma_{\text {sym }_{G}}^{2}
\end{array}\right]^{\top} .
$$

Therefore, for any values of $t_{1}, t_{2}, \tau_{1}$ and $\tau_{2}$, the diagonals of matrices $\boldsymbol{R}_{u u}\left(t_{1}, \tau_{1}\right)$ and $\boldsymbol{R}_{u u}\left(t_{2}, \tau_{2}\right)$ are simply scaled versions of each other. Consequently, this will also be the case for the diagonals of matrices $\Delta \boldsymbol{R}_{u \mathfrak{u}}^{\left(p_{1}, p_{2}, q\right)} \forall\left\{p_{1}, p_{2}, q\right\}$ in (20), implying that $\operatorname{rank}\left\{\left[\begin{array}{ll}\boldsymbol{m}_{g_{1}} & \boldsymbol{m}_{g_{2}}\end{array}\right]\right\}<2 \forall\left\{g_{1}, g_{2}\right\}$ and making identifiability of $Q$ impossible. This is a situation where the statistical diversity of the sources cannot be exploited due to the likeness of their distributions, and it is well know in such contexts that BSS cannot be performed using only second-order statistics (SOS).

It must be understood from such considerations that our approach cannot apply in the case of synchronous signals when identical pulse shaping functions are considered. However, in a general asynchronous scenario where it is plausible to assume that $\left\{\tau_{g}\right\}_{g=1}^{G} \sim$ $\mathcal{U}(0, T)$, a situation where $\tau_{g}=\tau_{0} \forall g$ (or generally any circumstance of conditions on $\left\{\tau_{g}\right\}_{g=1}^{G}$ implying that $\left.\operatorname{NAP}_{g}(t, \tau)=\operatorname{NAP}(t, \tau) \forall g\right)$ has a theoretically nil probability of occurrence. Nonetheless, there would still exist a non-zero probability that situations of closely distributed source signals (i.e. closely identical NAPs) be encountered since no control can be exerted on $\left\{\tau_{g}\right\}_{g=1}^{G}$ (i.e. without synchronization). A study of estimation performance in such unfavorable conditions is presented in Section 5.5.

In order to further enhance autocorrelation diversity such that it does not only depend on $\left\{\tau_{g}\right\}_{g=1}^{G}$, it will be assumed that each source signal in (6) is modulated using one among a set of distinct predefined pulse shaping functions (i.e. $p_{g}(t) \in\left\{\mathrm{p}_{s}(t)\right\}_{s=1}^{S} \forall g$ ) at each transmission block with no particular order or preference, and in an independent fashion for each user. This operation is accomplished on the mobile side only without requiring the use of synchronization signals and can be easily implemented by changing coefficients of a digital FIR pulse shaping filter from a set of predefined values stored in memory ${ }^{10}$. Considering that such set of coefficients be chosen randomly for each user from a finite set of possibilities, i.e. $\left\{\mathrm{p}_{s}(t)\right\}_{s=1}^{S<\infty}$, there would still exist a probability that $p_{g}(t)=p(t) \forall g$, and that an unfortunate circumstance such as $\tau_{g} \approx \tau_{0} \forall g$ results in creating unfavorable identifiability conditions. However, such an occurrence will be much less likely to be observed than if identical pulse shaping functions were to be used for all users. The overall operation thus contributes to enhance autocorrelation diversity, but is not a necessary requirement for application of

\footnotetext{
${ }^{10} \mathrm{By}$ changing the coefficients without affecting the number of symbol taps of a digital filter, the width of the transmit pulse shaping function remains constant and the operation brings literally no increase in hardware/implementation complexity.
} 
the algorithm considering that, as previously stated, the probability of occurrence of a natural event implying that $\operatorname{NAP}_{g}(t, \tau)=\operatorname{NAP}(t, \tau) \forall g$ is theoretically nil.

Finally, let us now resume the most important assumptions made throughout the paper, which, in a similar vein, are also general requirements for identifiability.

- A narrowband signal context with respect to the array dimensions. This allows the time domain received signal vector to be expressed in a compact matrix form such as (1).

- A time-invariant channel. This important assumption implies that matrix $\boldsymbol{B}$ remains constant over time (implying slow or nil fading), and is a necessary condition for BSS algorithms relying statistical estimates obtained by time averages.

- There is negligible delay spread. This allows the received source signals $u_{g}(t) \forall g \in\{1,2, \ldots, G\}$ to be modeled as independent random processes, and is necessary so that $\boldsymbol{R}_{u u}(t, \tau)$ be diagonal in (16).

- Signals are transmitted from the mobiles to the receiving array in a baud-asynchronous fashion where, for the duration of the observation period, $\tau_{g} \sim \mathcal{U}(0, T) \forall g$ where parameters $\left\{\tau_{g}\right\}_{g=1}^{G}$ are independent of each other. Such asynchronous conditions are naturally likely to arise in practical scenario, but we recall that our estimation principle cannot apply under synchronous conditions $\left(\tau_{g}=\tau_{0} \forall g\right)$ if identical pulse shaping functions are considered for all users $\left(p_{g}(t)=p(t) \forall g\right)$.

- Matrix $\boldsymbol{B} \in \mathbb{C}^{N \times G}$ has full column rank (which also implies that $G \leqslant N$ ). This is a necessary condition to allow its identifiability via JD.

- The noise vector $\boldsymbol{n}(t)$ is a stationary random process, or at least cyclostationary in $t$ with period $T /(a U)$ where $a \geqslant 1$ is an integer. This property is essential to obtain noise-free differential autocorrelation matrices $\Delta \boldsymbol{R}_{x x}^{\left(p_{1}, p_{2}, q\right)}$ in (20) which provide the necessary structure for a subsequent JD process.

\section{Simulation Results}

This section presents various simulation results intended to illustrate the performance behaviour of the proposed estimation algorithm in a broad variety of scenarios. It will be shown how by the sole use of SOS, blind identification of $\boldsymbol{B}$ can be achieved based on the DTM construction principle of eq. (20) using standard AJD techniques. The advantages of the latter principle will also be evidenced in cases of complex noise statistical distributions when a maximum number of users $G_{\max }=N$ is considered.

In essence, the algorithm proceeds in four main steps:

1) Given an integer oversampling factor $U$, compute $\boldsymbol{R}_{x x}^{(p, q)}$ for all $p^{\prime}$ s and $q^{\prime}$ s in (18).

2) Form the set of DTM from (20), (28) and (26).

3) Estimate $Q$ by perform AJD on (28) using an appropriate existing algorithm.

4) Estimate $\boldsymbol{B}$ from (30).
Considering an observation time of $P$ symbol periods, each of matrices $\boldsymbol{R}_{x x}^{(p, q)}$ in (18) are estimated by (refer to Fig. 5):

$$
\begin{gathered}
\hat{\boldsymbol{R}}_{x x}^{(p, q)}=\frac{1}{k_{\max }+1} \sum_{k=0}^{k_{\max }} x_{U k+p} x_{U k+p+q}^{\dagger}, \\
k_{\max }=\left\lfloor\frac{U P-p-q}{U}\right\rfloor .
\end{gathered}
$$

For simplicity, the received pulse shaping functions $p_{g}(t) \forall g$ are considered to be ideal windowed (rectangular) RC pulse functions of width $W=6 T$ (see eqs. (A.5) and (A.11) in Appendix) for all experiments. As explained in Section 4.3, each source signal is transmitted using one among a set of distinct predefined pulse shaping functions (i.e. $p_{g}(t) \in\left\{\mathrm{p}_{s}(t)\right\}_{s=1}^{S}$ ). These distinct functions will be generated in the present case considering a change of parameter $\beta$, leading to the definition of a vector $\beta=\left[\begin{array}{llll}\beta_{1} & \beta_{2} & \ldots & \beta_{G}\end{array}\right]^{\top}$ representing the rolloff factors of each corresponding source signal. Likewise we also define a vector $\tau=\left[\begin{array}{llll}\tau_{1} & \tau_{2} & \ldots & \tau_{G}\end{array}\right]^{\top}$ representing the corresponding asynchronous delays.

The performance index used in all experiments is a similar version of the global rejection level (GRL) introduced in [17]. In its original form we have:

$$
E\{\mathrm{GRL}\}=\sum_{p \neq q} E\left\{\left|\left[\hat{\boldsymbol{B}}^{\#} \boldsymbol{B}\right]_{p q}\right|^{2}\right\} \geqslant 0 .
$$

The definition is based on the idea that a close estimate $\hat{\boldsymbol{B}}$ of $\boldsymbol{B}$ implies that $\hat{\boldsymbol{B}}^{\#} \boldsymbol{B} \approx \boldsymbol{I}$, which in turn yields GRL $\approx 0$. This formulation, however, implies that larger column-sized matrices generate higher GRLs than smaller sized ones for equivalent quality estimates (say, the distribution of $\left|\left[\hat{\boldsymbol{B}}^{\#} \boldsymbol{B}\right]_{p q}\right|^{2}$ for $p \neq q$ ) since the net sum of all anti-diagonal elements is considered. In order to compare the estimation performance of differently sized channel matrix on a same basis, we define a modified and averaged version of the GRL inspired from [24] such that:

$$
\begin{aligned}
\mathrm{GRL}= & {\left[\sum_{p=1}^{G}\left(\sum_{q=1}^{G} \frac{H_{p q}}{\max _{k}\left(H_{p k}\right)}-1\right)+\right.} \\
& \left.\sum_{q=1}^{G}\left(\sum_{p=1}^{G} \frac{H_{p q}}{\max _{k}\left(H_{k q}\right)}-1\right)\right] / G^{2}, \\
H_{p q}= & \left|\left[\hat{\boldsymbol{B}}^{\#} \boldsymbol{B}\right]_{p q}\right|^{2}, \boldsymbol{B} \in \mathbb{C}^{N \times G}, N \geqslant G .
\end{aligned}
$$

This new performance index goes to zero only if $\hat{\boldsymbol{B}}$ is essentially equal to $\boldsymbol{B}$ (i.e. if $\hat{\boldsymbol{B}}=\boldsymbol{B Z}$ where $\boldsymbol{Z}$ is a scale and column permutation matrix). The computation averaged over all $G^{2}$ elements of $\hat{\boldsymbol{B}}^{\#} \boldsymbol{B}$ now provides a better comparison basis ${ }^{11}$, and for this reason eq. (48) will be considered for all experiments.

The theoretical exact power definition of a received source signal is:

$$
P_{g}=\lim _{T \rightarrow \infty} \frac{1}{T} \int_{-T / 2}^{T / 2}\left|u_{g}(t)\right|^{2} d t,
$$

\footnotetext{
${ }^{11}$ Normalized performance criterions similar to (48) were also considered in [26].
} 
and depends on the variance $\sigma_{\text {sym }_{g}}^{2}$ of the transmitted symbol sequence as well as the pulse shaping function $p_{g}(t)$, as shown in Appendix. In all simulations however, we will more conveniently refer to the power of a received signal $u_{g}(t)$ as the variance $\sigma_{\text {sym }}^{2}$ of its corresponding symbol sequence. Such a designation (although inexact) will allow to better compare the estimation performance of differently distributed signals through the use of different pulse shaping functions, and will not impact on the generality of the observations to be made.

For all experiments, a SNR measure is defined as the ratio of mean source symbol power to mean noise power over all elements, that is:

$$
\mathrm{SNR}=\frac{N \sum_{g=1}^{G} \sigma_{\mathrm{sym}_{g}}^{2}}{G \sum_{p=1}^{N} \sigma_{n_{p}}^{2}} .
$$

However, since equal source and noise powers will be considered in all cases, this expression reduces to $\mathrm{SNR}=\sigma_{\mathrm{sym}}^{2} / \sigma_{n}^{2}$. In experiments 1 to 5 , a temporally and spatially white Gaussian noise will be considered to emphasize performance behaviour with respect to signal related aspects. A differently distributed noise (but sill Gaussian) will be considered in experiment 6 .

Note also that in order to more conveniently evaluate the performance index of differently parametrized signals in various contexts, it will be assumed that $G$ is known. In practice however, an estimate of $G$ can be obtained by application of the well known AIC [60] or MDL [61] detection criteria on the singular values of (25).

Note finally that a parameter $\mathcal{S}$ will be used in all experiments to denote the number of simulation runs used for averaging operations.

\subsection{Experiment 1: Performance Comparison of Different AJD Algorithms}

This first experiment is intended to compare the performance of different AJD algorithms in a same signal context. The literature provides a vast assortment of algorithms capable of solving the JD problem of eq. (28). In this section however, the following four algorithms will be considered:

- CVFFDIAG [25]. This algorithm represents an adaptation of the popular FFDIAG [29] to complexvalued problems. The authors propose a lowcomplexity iterative scheme having good performance and a relative ease of implementation.

- U-WEDGE [23]. This algorithm presents an interesting iterative approach based on exhaustive diagonalization using Gaussian iterations. It is notably popular in the literature and proves directly compatible with the set of target matrices in (28).

- s-BIA [24]. This AJD algorithm implements an original bi-iterative process inspired from ACDC [27]. The authors provide a comprehensive literature review along with various simulation results demonstrating the effectiveness of the proposed method.
- IDIEM [26]. This recent work presents an interesting direct (noniterative) solution to the AJD problem of a set of complex eigenmatrices. The algorithm has a low computational complexity and provides closed-form approximate solutions for the direct least-squares optimization criterion.

In this experiment, an array of $N=4$ elements is considered along with $G=2$ impinging BPSK-modulated signals and an upsampling factor $U=2$. Fixed parameters $\boldsymbol{\beta}=\left[\begin{array}{ll}0.2 & 0.4\end{array}\right]^{\top}$ and $\boldsymbol{\tau}=T\left[\begin{array}{ll}0.1 & 0.5\end{array}\right]^{\top}$ are also considered. Fig. 7 displays the overall results for each algorithm considering both the full and "more representative" set of target matrices according to (28) and (39). Note however that the IDIEM algorithm already implements a computation of a "more representative" set of target matrices (also inspired from [58]), and for this reason only the full set of target matrices is considered in its case. Each point of the curves in Fig. 7 is generated from $\mathcal{S}=500$ simulation runs considering block lengths of $P=2000$ symbol periods. Discontinuities represent a non convergence of the estimated solution for the considered algorithm, which happens as a joint diagonalizer estimate $\hat{V}$ becomes close to singular, and is detected using a threshold on the norm of $\hat{Q}$ in (30).

The first three graphs are generated considering fixed channel matrices (but different ones in each three cases), which is meant to better appreciate the behaviour of each algorithm in different numerical contexts. In (a) specifically, it can be seen that even at high SNRs CVFFDIAG, s-BIA and IDIEM converge to a non-optimal solution ${ }^{12}$, whereas U-WEDGE still achieves good performance. It can also be seen that the use of the "more representative" set of target matrices generally worsens performance in all cases, and at a higher degree at low SNRs. This somewhat counterintuitive observation can be explained from the fact that, although the use of eq. (39) significantly reduces the complexity of the AJD problem, the effective set reduction operation, from the standpoint of information theory, represents a simple loss of information. Although it was mentioned in [58] that the two "more representative" matrices could be either used for direct AJD or as an initial guess for a subsequent iterative algorithm, a general approach aiming at restricting the number of target matrices will always be subject to such a drawback. As observed in Fig. 7, the operation becomes more beneficial however as the SNR or more specifically the observation time PT increases, implying that the initial set of target matrices becomes closer to its ideal eigenstructure, but otherwise is not likely to bring improvement in performance.

Fig. 7 also shows that U-WEDGE clearly outperforms other algorithms for all SNR values in a consistent manner. This overall behaviour is confirmed in case (d) where matrix $\boldsymbol{B}$ is given a random value at each simulation run with $B_{p q} \sim \mathcal{N}(0,1)+j \mathcal{N}(0,1)$. This emphasises the fact that the choice of an appropriate AJD algorithm is an important factor in maximizing the

\footnotetext{
${ }^{12}$ Convergence of AJD algorithms toward local extrema is a well known problem in the literature. See [27, 30].
} 


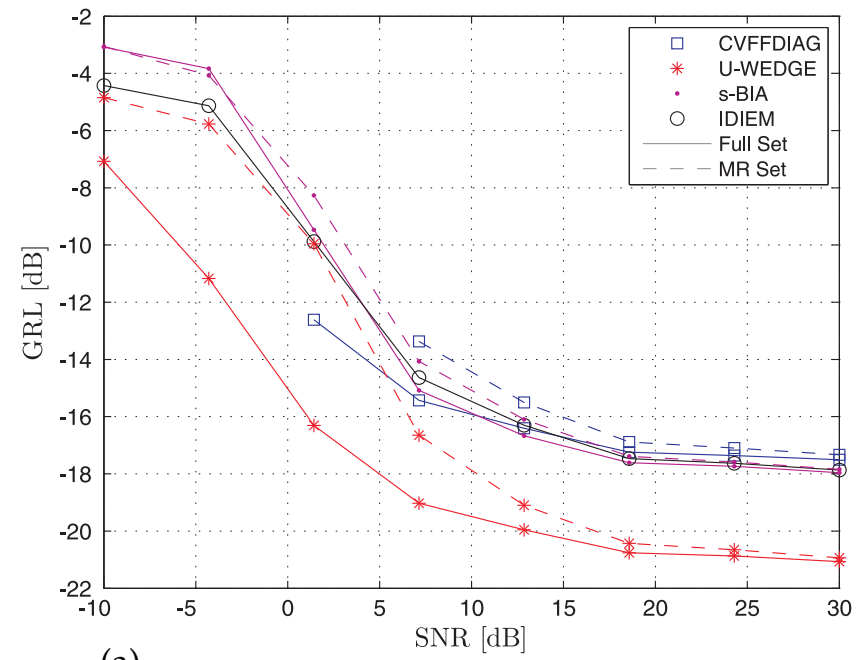

(a)

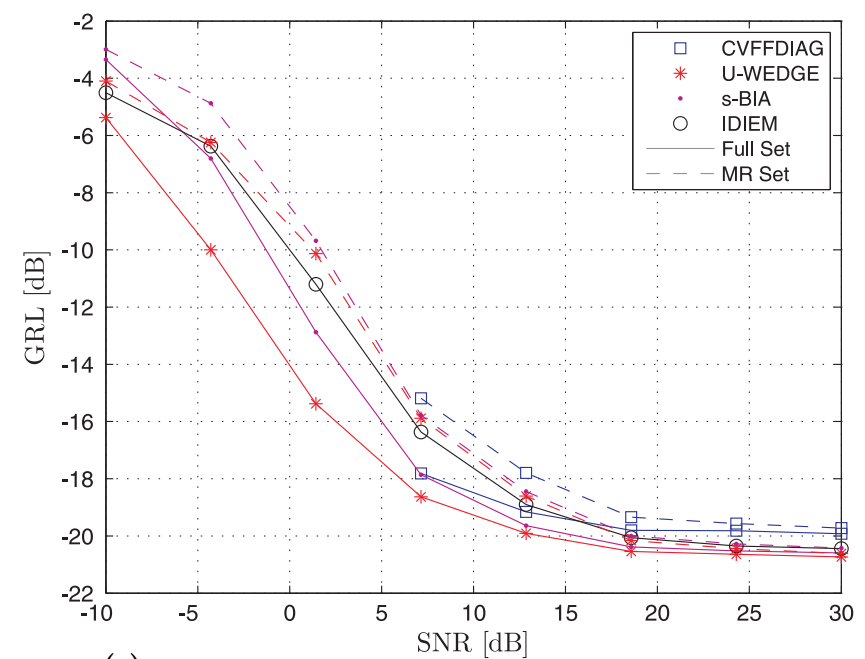

(c)

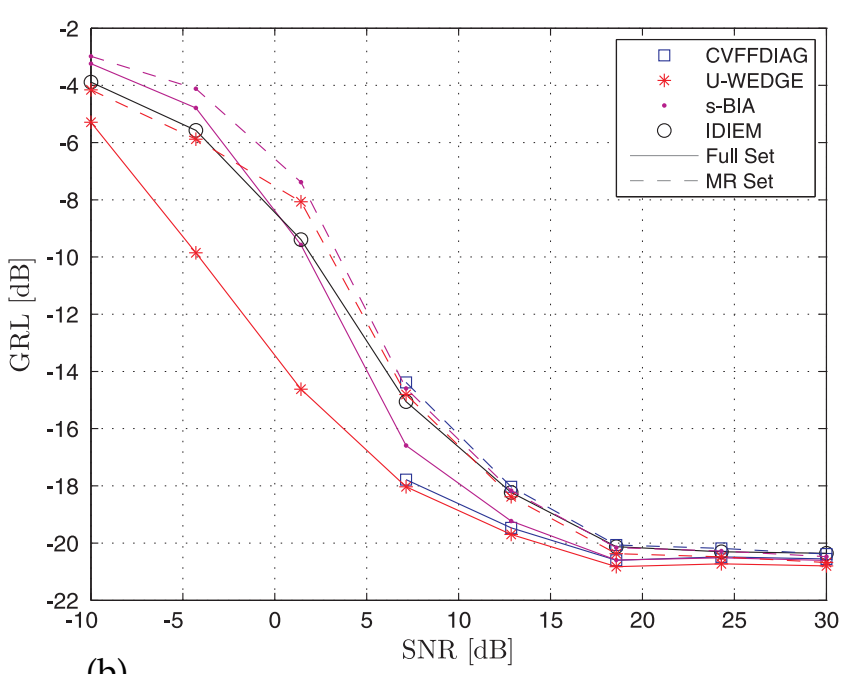

(b)

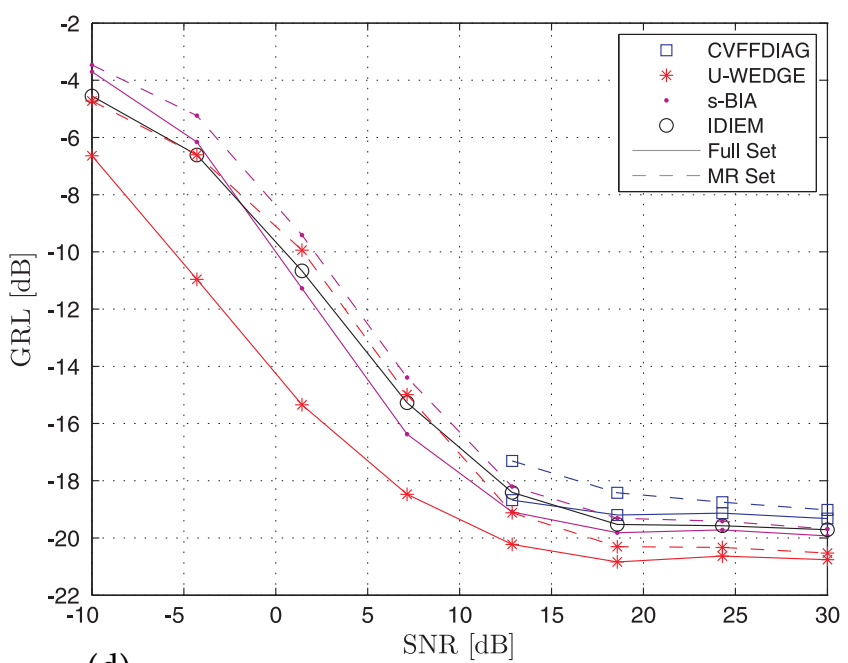

(d)

Figure 7. Results of experiment 1 . Three different fixed channel matrices are considered in (a), (b) and (c). In (d), elements of $B$ are randomly generated at each simulation run from a complex Gaussian distribution.

output estimation performance. Other experiments and simulations have also shown that U-WEDGE provides the best overall performance among other algorithms, and for this reason it will be retained for the next experiments.

\subsection{Experiment 2: Effect of Signal Constellations}

It was shown in Section 4.3 that in order to achieve blind identification, at least two source signals must have different NAPs. It can be seen from eq. (A.4) that a source NAP is independent of the symbol distribution. Hence this suggests that differently modulated signals (via different symbol distributions) should yield identical estimation performance if the same pulse shaping functions are used, even if from a statistical point of view, the signals are differently distributed. This experiment is meant to exemplify this behaviour. Fig. 8 displays simulation results obtained considering $N=4, G=3, U=3, \beta=\left[\begin{array}{lll}0.4 & 0.4 & 0.4\end{array}\right]^{\top}$, $\tau=T\left[\begin{array}{lll}0.4 & 0.8 & 0.5\end{array}\right]^{\top}, P=2000$ and $\mathcal{S}=500$. Elements of $\boldsymbol{B}$ are also given random values at each simulation run as in experiment 1 . We observe that identical performance is achieved in all cases, thus confirming that signal constellations have no effect on the average quality of estimate.

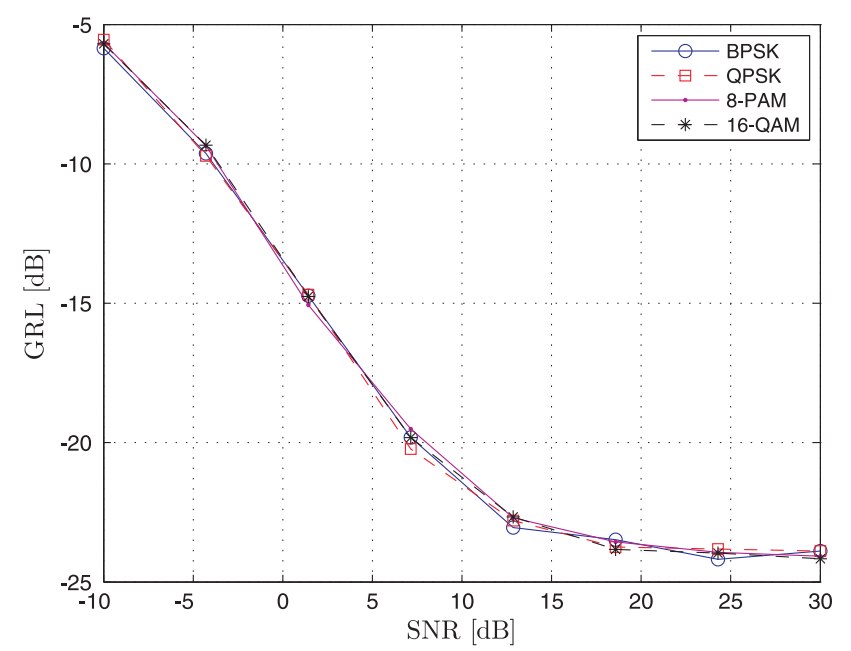

Figure 8. Results of experiment 2. Estimation performance considering source signals having different constellations. AJD is performed using U-WEDGE. 


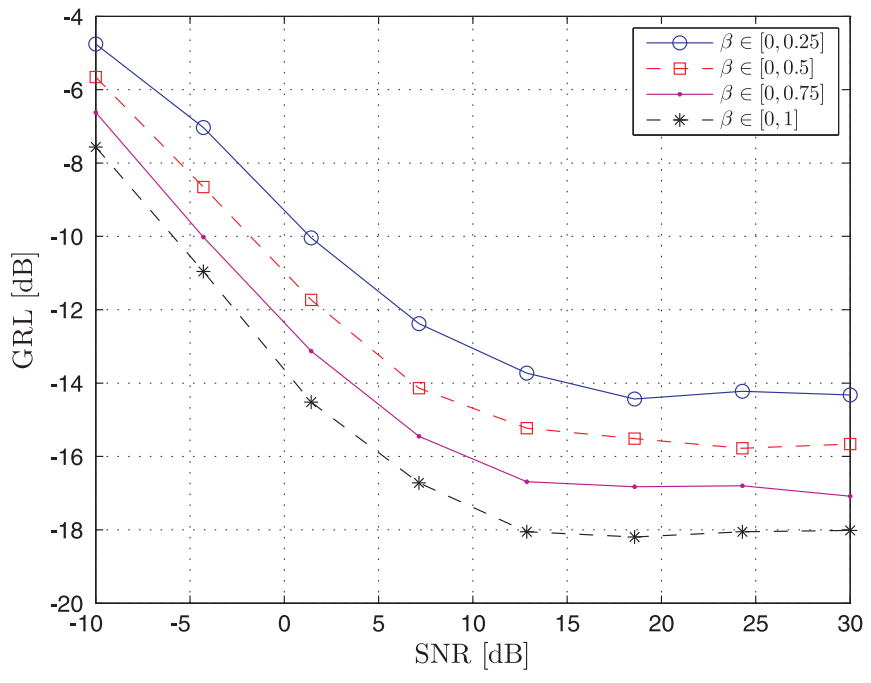

Figure 9. Results of experiment 3. Performance comparison for source signals having different pulse shaping functions. AJD is performed using U- WEDGE.

\subsection{Experiment 3: Effect of Pulse Shaping Functions}

Autocorrelation diversity can be exploited if the received signals have different NAPs, which according to eq. (A.4) are a sole function of the source pulse shaping functions. This experiment is intended to exemplify the dependency of estimation performance on this particular signal aspect. More particularly, Fig. 4 shows that as $\beta$ decreases in (A.11), a source NAP amplitude $\left(\max _{t}(\mathrm{NAP}(t, \tau))-\min _{t}(\operatorname{NAP}(t, \tau))\right)$ also decreases, hence impacting negatively on autocorrelation diversity. The tendency is particularly visible on the graphs of the first row of the same figure for large window lengths. Fig. 9 displays simulation results obtained for signals having different pulse shaping functions, which is modeled via changes in distribution of parameter $\beta$. An array of $N=6$ elements is considered, along with $G=316$-QAM modulated signals and an upsampling factor $U=3$. In this experiment, $B, \beta$ and $\tau$ are given random values at each simulation run such that $B_{p q} \sim \mathcal{N}(0,1)+j \mathcal{N}(0,1), \beta_{g} \sim \mathcal{U}\left(0, \beta_{\max }\right)$ and $\tau_{g} \sim \mathcal{U}(0, T)$. Calculations are performed considering $P=2000$ symbol periods and $\mathcal{S}=1500$ trials. The performance dissimilarity for each set of parameter distribution is obvious. As intuitively expected, lower values of $\beta$ decrease overall performance by providing less diversity between elements of $\operatorname{diag}\left\{\boldsymbol{R}_{u u}^{(p, q)}\right\}$ in (18), which in turn impacts negatively in (20). Similar results could also have been obtained considering identical distribution of parameter $\beta$ in all cases, but different distribution of window length $W$. This experiment emphasises the fact that the nature of the source pulse shaping functions plays a significant role in optimizing estimation performance.

\subsection{Experiment 4: Influence of the Number of the Target Matrices}

This interesting experiment is meant to investigate the effect on estimation performance of the total number of target matrices considered for the AJD problem

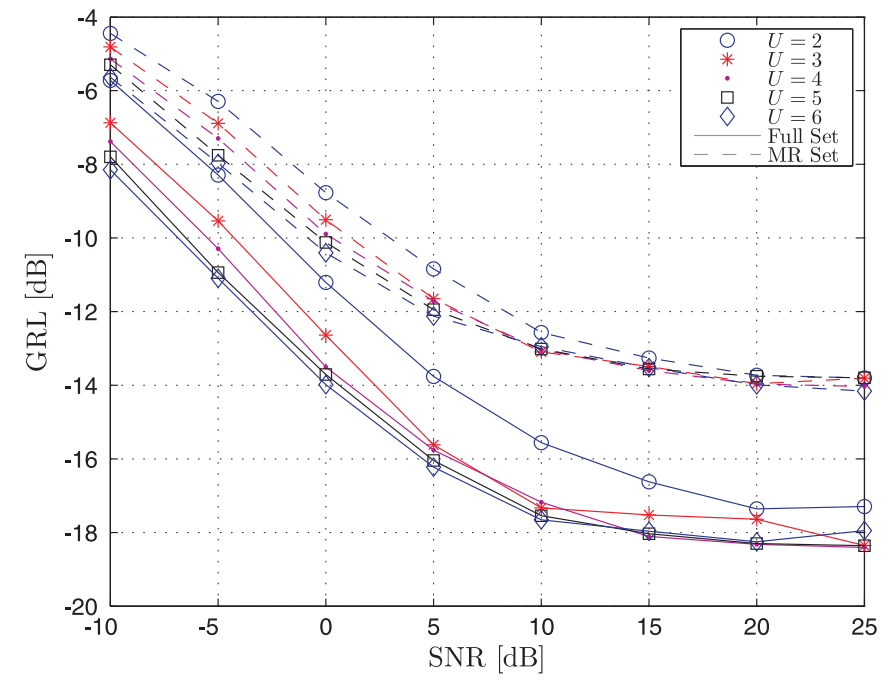

Figure 10. Results of experiment 4. Effect of upsampling factor $U$ on estimation performance. For each $U$ the number of target matrices is given by (22). AJD is performed using U-WEDGE.

in eq. (28). The number of target matrices $D$ is set by varying $U$ according to (22). In this simulation, a five-element array is considered with $G=3$ impinging QPSK signals. $P=2000$ symbol periods are also used for calculation of estimated autocorrelation matrices, and results are averaged over $\mathcal{S}=2000$ simulation runs, where at each time $B_{p q} \sim \mathcal{N}(0,1)+j \mathcal{N}(0,1)$, $\beta_{g} \sim \mathcal{U}(0,1)$ and $\tau_{g} \sim \mathcal{U}(0, T)$. Fig. 10 displays the experiment results considering the values of $U$ and $D$ in Table I. For comparison, curves associated with the "more representative" set of target matrices have also been included. As in experiment 1 , it can be seen that the set reduction consistently worsens performance over the considered SNR range, despite bringing significant gain in computational complexity (for the AJD problem) as $U$ increases. Interestingly, it can be observed that an increase in the number of target matrices brings consistent improvement in performance (considering the full set), especially at low SNRs. This phenomenon is attributable to a better exploitation of signal statistics via multiple autocorrelation evaluations, and was also the main motivation in the development of [17]. However the gain in Fig. 10 reaches a finite limit as $U$ increases and comes at the price of significant increases in computational complexity. At high SNRs, a less important gain is obtained for high numbers of target matrices, which could be intuitively predicted especially as $P$ increases.

\subsection{Experiment 5: Effect of Close Distributions}

This experiment is intended to examine the performance of the estimation procedure in a context of closely distributed signals. More particularly, we refer to closely distributed signals as signals having similar NAPs, where, to an extent, estimation of $\boldsymbol{B}$ via JD of (28) cannot be performed (see Section 4.3). In experiment 1 and 2, fixed parameters $\beta$ and $\tau$ were considered at all simulation runs and SNRs, and ensured that sources have different NAPs. In experiment 3 and 4, a more 
complete set of test conditions was considered where $\beta$ and $\tau$ were uniformly generated in continuous intervals at each simulation run. Overall estimation performance thus encompassed cases of closely distributed signals, but their effect could not be emphasized. In this experiment, in order to better examine the behaviour of estimation performance in this context, we consider a scenario where $\beta$ and $\tau$ are fixed at each simulation run but are progressively given values according to a parameter $\kappa$. For $\kappa=0$, the values of $\beta$ and $\tau$ are imposed such that the sources have different NAPs. For $\kappa=1$, we have $\beta_{g}=\beta \forall g$ and $\tau_{g}=\tau \forall g$, implying that the sources be identically modulated and perfectly synchronous, thus having identical NAPs.

Simulations are performed considering an array of $N=4$ elements, $G=2$ BPSK-modulated signals and an upsampling factor $U=3$. Three sets of parameter values for $\beta$ and $\tau$ are considered:

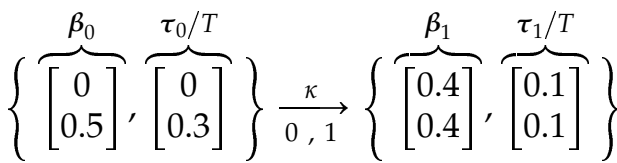

$$
\begin{aligned}
& \left\{\left[\begin{array}{l}
0.2 \\
0.5
\end{array}\right],\left[\begin{array}{l}
0.6 \\
0.9
\end{array}\right]\right\} \underset{0,1}{\stackrel{\kappa}{\longrightarrow}}\left\{\left[\begin{array}{l}
0.3 \\
0.3
\end{array}\right],\left[\begin{array}{l}
0.5 \\
0.5
\end{array}\right]\right\} \\
& \left\{\left[\begin{array}{l}
0 \\
1
\end{array}\right],\left[\begin{array}{l}
0.4 \\
0.9
\end{array}\right]\right\} \underset{0,1}{\stackrel{\kappa}{\longrightarrow}}\left\{\left[\begin{array}{l}
0.5 \\
0.5
\end{array}\right],\left[\begin{array}{l}
0.5 \\
0.5
\end{array}\right]\right\}
\end{aligned}
$$

Fig. 11 (a) displays the performance curves obtained for the three sets considering $P=2000$ symbol periods, $\mathcal{S}=1000$ simulation runs and a SNR of $10 \mathrm{~dB}$. In each case good performance is obtained for almost all values of $\kappa$ up to about 0.9 . As $\kappa$ increases, optimality is reached in the statistical distribution of the sources from points $\left\{\boldsymbol{\beta}_{0}, \boldsymbol{\tau}_{0}\right\}$ to $\left\{\boldsymbol{\beta}_{1}, \boldsymbol{\tau}_{1}\right\}$, which explains the presence of GRL minima for the three curves at different locations. For $\kappa=1$, sources have identical NAPs and the JD of (28) cannot be performed. The value of about $-4 \mathrm{~dB}$ observed at this point has no particular meaning with regard to estimation performance.

The case of received source signals having identical NAPs has a zero probability of occurrence considering the time-continuous nature of $\tau_{g} \forall g$ and the use of appropriate pulse shaping functions. However, situations of closely distributed received signals are likely to be encountered with a non-zero probability, and estimation performance may therefore be reduced. Fig. 11 (b) displays the performance curves obtained at $\kappa=0 . \overline{8}$ considering the sets of parameters of eq. (51) as a function of the number of symbol periods $P$. As expected, the results show that good performance can still be achieved in this context by considering larger observation times.

\subsection{Experiment 6: Performance Assessment in Complex Noise Environments}

This last experiment is intended to underline the advantages of the DTM construction principle explained in Section 4. The array's degree of freedom is now exploited to its full extent by considering multiple

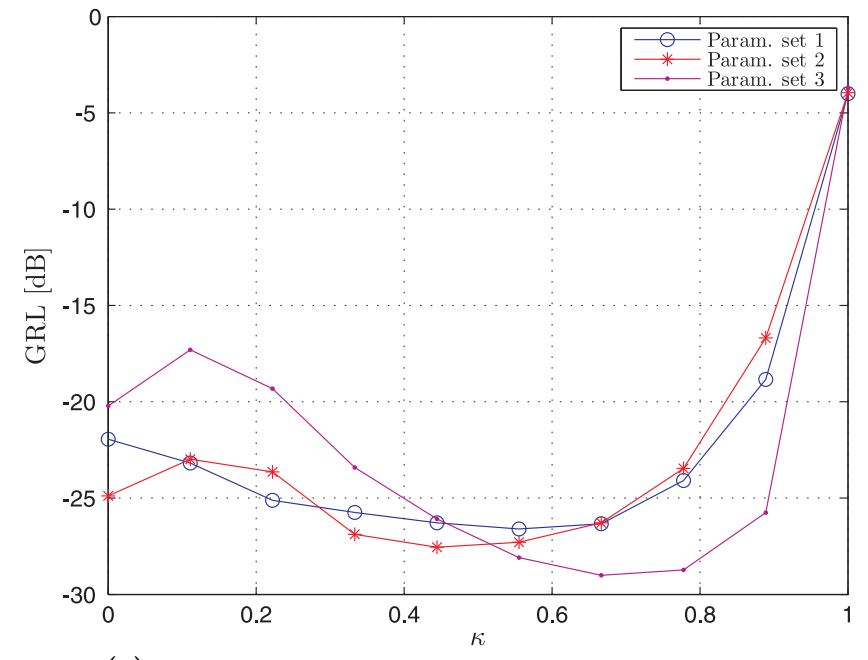

(a)

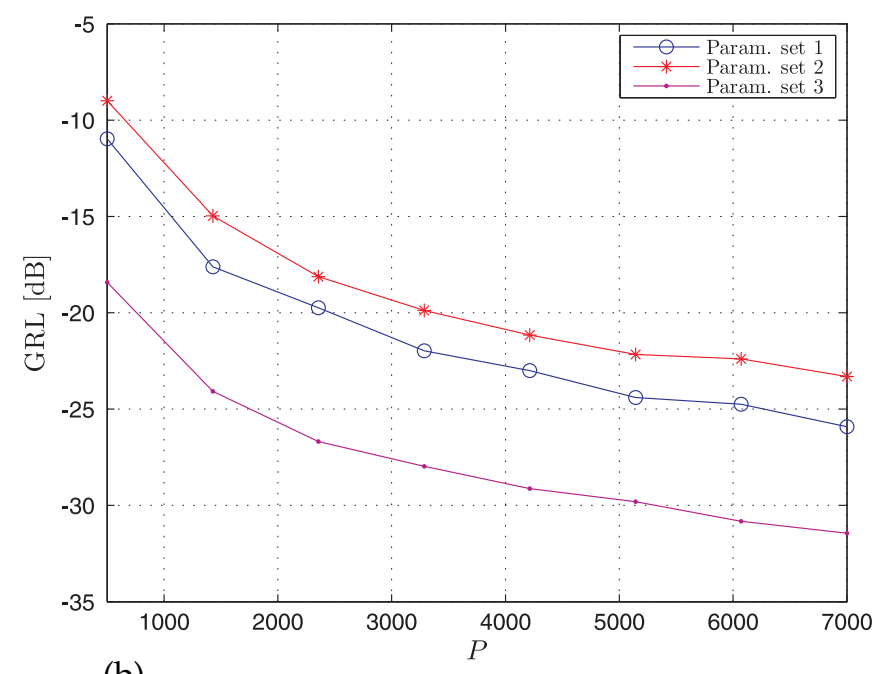

(b)

Figure 11. Results of experiment 5. (a): Estimation performance as the NAPs of two received signals progressively becomes identical. (b): Effect of the number of symbol periods on overall performance for $\kappa=0 . \overline{8}$ in (a). AJD is performed using U-WEDGE.

instances of scenarios for which $N=G$. A temporally Gaussian colored noise having the bell shape of Fig. 12 is also considered with a SNR measurement given by (50). The noise is however spatially white. In such conditions, noise variance estimation cannot be performed by performing an eigenvalue analysis of any zero-lag autocorrelation matrix estimate. Moreover, non-zero-lag autocorrelation matrices will not possess the required eigenstructure for AJD unless a sufficiently large time lag is considered (approximately 60T/U in the case of Fig. 12). Approaches where estimation of the mixing matrix is directly performed from the set of autocorrelation matrix estimates as in [18-21] can therefore not be applied. In this simulation, 16-QAM signals are considered with an upsampling factor $U=4$. Fig. 13 presents the performance curves associated with different values of $N=G$ as a function of $P$ considering a mean SNR of $-5 \mathrm{~dB}$. The number of simulation runs is adjusted linearly in the interval from $\mathcal{S}=4000$ to $\mathcal{S}=3000$, where at each time $B_{p q} \sim \mathcal{N}(0,1)+j \mathcal{N}(0,1)$, $\beta_{g} \sim \mathcal{U}(0,1)$ and $\tau_{g} \sim \mathcal{U}(0, T)$. It can be observed 


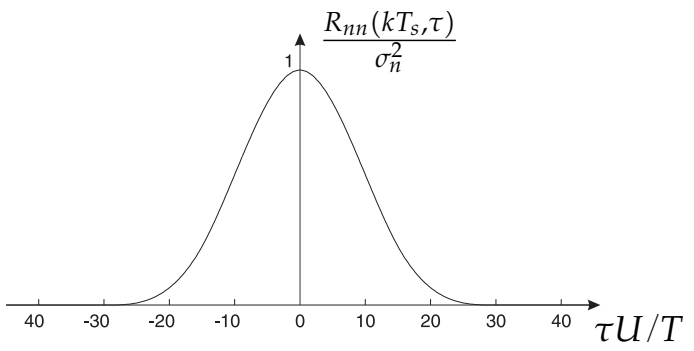

Figure 12. Noise autocorrelation function at each sampling instant $t_{k}=k T_{s}$ used in experiment 6 .

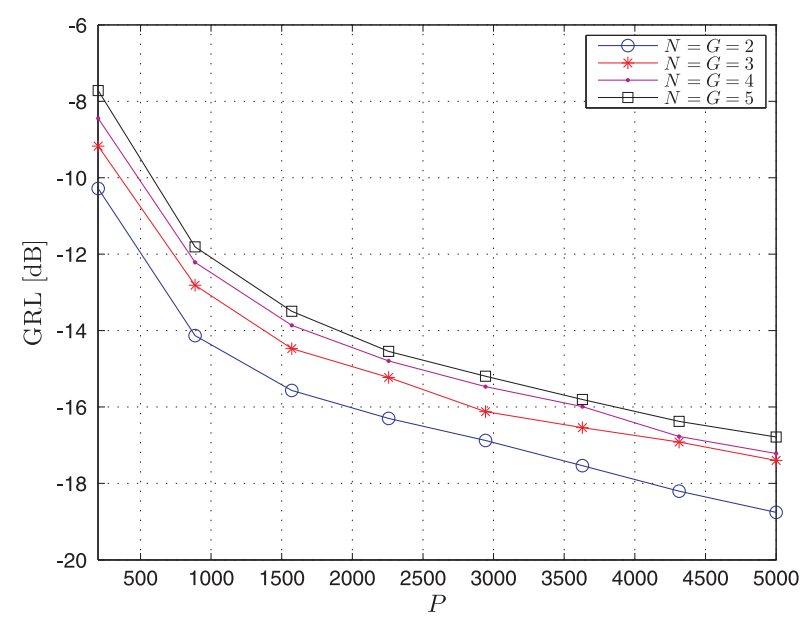

Figure 13. Results of experiment 6. Estimation performance as a function of the number of symbol periods for different values of $N$ and $G$ considering a temporally colored noise of $-5 \mathrm{~dB}$. AJD is performed using U-WEDGE.

that better performance is achieved for smaller number of sources, which is attributable to smaller average norms of matrices $\hat{\boldsymbol{R}}_{u u}(t, \tau)$ and from the AJD operation itself. As $P$ increases, the GRL decreases in all cases and confirms the effectiveness of the noise cancellation process of eq. (20).

\section{FUTURE WORKS}

We have shown how the cyclostationary nature of communication signals in a general asynchronous context could be exploited to perform BSS considering the JD of a set of differential second-order autocorrelation matrices. However we have also shown in Section 4.3 that such an approach may be subject to irregular estimation performance given the random character of $\left\{\tau_{g}\right\}_{g=1}^{G}$ in (6), implying unpredictable statistical distribution of the sources at each observation period (generally) and giving rise to potential unfavorable estimation conditions (similarity of the NAPs). Although identifiability of $\boldsymbol{B}$ to within a scale and permutation factor is still possible in such contexts provided that a sufficiently large number of sample is available, an interesting comparison could be made between estimation performance of the proposed method and that of a higherorder algorithm such as JADE [16] (or an equivalent algorithm considering a set of DTM as in (28)) possibly less prone to such performance variation. We wish to stress however that this change in statistical distribution of the sources due to unpredictable values of $\left\{\tau_{g}\right\}_{g=1}^{G}$ in (6) is an intrinsic property of asynchronous signals to which any algorithm would have to be subject to with a wide range of consequences on estimation performance depending on the type of processing at work.

Derivation of Cramér-Rao bounds considering either fixed or uniformly distributed values of $\left\{\tau_{g}\right\}_{g=1}^{G}$ for specific families of pulse shaping functions would also constitute a yet complex but very interesting problem proving instrumental for performance benchmarking. Similarly, obtention of a lower bound on the GRL in terms of general estimation parameters such as $U$ in experiment 4 would also prove useful. For length concerns, such analysis have not been included in the paper.

In another vein, an interesting parallel was made in Section 2.2.2 regarding expression of the received signal vector considering either a FIR-MIMO-based modeling or a traditional array processing signal model where the source vector directly encompasses the time-continuous expression of the source signals. The main point of this discussion was to underline the fact that although the use of a FIR-MIMO-based modeling is a convenient way of expressing the observable outputs in terms of the transmitted symbol sequences of each user, it implies having to deal with generally different channel matrices if oversampling techniques are to be exploited. On the other hand, the use of the classical signal model (4) implies that $\boldsymbol{B}$ remains constant with sampling phase but isn't as practical to recover the transmitted symbol sequence of each user. To this end however, an approach as considered in [36] may be employed where source symbol values for each user are recovered via beamforming ${ }^{13}$ using an optimal weight vector of the form:

$$
\boldsymbol{w}_{\mathrm{opt}_{g}}=c \boldsymbol{R}_{x x}^{-1} \boldsymbol{b}_{g}, g \in\{1,2, \ldots, G\},
$$

where $\boldsymbol{R}_{x x}$ is the spatial autocorrelation matrix (obtained in the synchronous conditions of Fig. 1 (a)) and where $c$ is an adaptive constant. Estimates of the source signals are then obtained as:

$$
\hat{u}_{g}\left(t_{k}\right)=w_{\text {opt }_{g}}^{\dagger} x_{k} \forall g \in\{1,2, \ldots, G\},
$$

where $\left\{t_{k}\right\}_{k=1}^{K}$ are symbol instants identical since no oversampling is considered in [36]. The optimum weight vector (52) is well known in the literature for maximizing the signal-to-interference plus noise ratio (SINR). However its definition given a desired array response vector solely relies on the spatial autocorrelation matrix (see [35]), which is a sufficiently representative statistical measure of the observed signals only under stationary conditions. It is worth noting that the benefits of smart antenna techniques through the use of beamforming for example are typically promoted in a context of wireless communications involving cyclostationary signals. We recall from Section 3 that

\footnotetext{
${ }^{13}$ Note that synchronous signals and perfect symbol timing recovery are assumed in [36].
} 
$x\left(t_{k}\right)$ for $t_{k}=t_{0}+k T$ is a stationary random process, whereas $x(t)$ is rather cyclostationary in $t$ with period $T$. Hence a general zero-lag autocorrelation matrix $\boldsymbol{R}_{x x}(t)=E\left\{x(t) \boldsymbol{x}^{\dagger}(t)\right\}$ depends on $t$, or equivalently on the time reference $t_{0}$ (or phase) of a set of sampling instants taken at the baud rate. In such conditions, an optimum weight vector could be obtained at any arbitrary time $t=t_{0}$ similarly to (52) such that:

$$
\boldsymbol{w}_{\text {opt }_{g}}\left(t_{0}\right)=c \boldsymbol{R}_{x x}^{-1}\left(t_{0}\right) \boldsymbol{b}_{g}, g \in\{1,2, \ldots, G\} .
$$

In the case of identically modulated and synchronous signals (i.e. $p_{g}(t)=p(t)$ and $\left.\tau_{g}=\tau_{0}\right\} \forall g$ as in Fig. $1(\mathrm{a}))$, any $\boldsymbol{w}_{\mathrm{opt}_{g}}\left(t_{0_{a}}\right)=\alpha_{a, b} \boldsymbol{w}_{\mathrm{opt}_{g}}\left(t_{0_{b}}\right)$ where $\alpha_{a, b}$ is a real constant since matrices $\boldsymbol{R}_{u u}(t, 0) \forall t$ are simply scaled versions of each other due to source signals having identical NAPs (see Section 3). An autocorrelation matrix $\boldsymbol{R}_{x x}\left(t_{0}, 0\right)$ may then be evaluated at any $t_{0}$ for obtention of an optimum weight vector to be used in (53). However the statistical distribution of the source signals $\left\{u_{g}\left(t_{k}\right)\right\}_{g=1}^{G}$ would indeed depend on $t_{0}$.

In a general asynchronous case, matrices $\boldsymbol{R}_{u u}(t, 0) \forall t$ are no longer scaled versions of each other and source signal estimates $u_{g}\left(t_{k}\right)$ cannot be optimally recovered from a single weight vector. Considering the set of zero-lag autocorrelation matrices $\left\{\boldsymbol{R}_{x x}^{(p, 0)}\right\}_{p=0}^{U-1}$ in (18), a distinct weight vector may then be computed for each sampling phase index $p$ such that:

$$
\begin{gathered}
\boldsymbol{w}_{\mathrm{opt}_{g}}^{(p)}=c\left(\boldsymbol{R}_{x x}^{(p, 0)}\right)^{-1} \boldsymbol{b}_{g}, \\
g \in\{1,2, \ldots, G\}, p \in\{0,1, \ldots, U-1\},
\end{gathered}
$$

and source signal estimates may then be obtained as:

$$
\begin{gathered}
\hat{u}_{g}^{(p)}\left(t_{k}\right)=\left(\boldsymbol{w}_{\mathrm{opt}_{g}}^{(p)}\right)^{\dagger} \boldsymbol{x}_{k}^{(p)} \\
\forall g \in\{1,2, \ldots, G\}, p \in\{0,1, \ldots, U-1\},
\end{gathered}
$$

where $\left\{x_{k}^{(p)}=x_{U k+p}\right\}_{k=1}^{K}$ is the set of output vector samples considered for evaluation of $\boldsymbol{R}_{x x}^{(p, 0)}$. An estimate of the $g$-th source signal sequence $\left\{u_{g}\left(t_{0}\right), u_{g}\left(t_{1}\right), \ldots\right\}$ could then be obtained from an interleaved reconstruction of its $U$ baud-sampled sequence estimates $\left\{\hat{u}_{g}^{(p)}\left(t_{k}\right)\right\}_{p=0}^{U-1}$ such that:

$$
\begin{gathered}
\left\{\hat{u}_{g}\left(t_{0}\right), \hat{u}_{g}\left(t_{1}\right), \ldots\right\}= \\
\left\{\hat{u}_{g}^{(0)}\left(t_{0}\right), \hat{u}_{g}^{(1)}\left(t_{0}\right), \ldots, \hat{u}_{g}^{(U-1)}\left(t_{0}\right), \hat{u}_{g}^{(0)}\left(t_{1}\right), \hat{u}_{g}^{(1)}\left(t_{1}\right), \ldots\right\},
\end{gathered}
$$

where in general $\hat{u}_{g}\left(t_{k}\right)=\hat{u}_{g}^{(k \% U)}\left(t_{[k / U]}\right)$ with '\%' denoting the modulo operator. The signal reconstruction procedure of eqs. (55), (56) and (57) is in essence identical to that of classical beamforming techniques, with the difference that a distinct weight vector is considered for each of $U$ baud-sampled sequences. This strategy is one among multiple ways in which estimation of the source signals $\left\{u_{g}\left(t_{k}\right)\right\}_{g=1}^{G}$ could be performed in a general asynchronous context upon estimation of $\boldsymbol{B}$ in (30). For example, more effective techniques could potentially be developed by also making use of matrices $\boldsymbol{R}_{x x}^{(p, q)} \forall\{p, q \neq 0\}$ in (18) since no time lag autocorrelation is exploited in (55). Interestingly, note that this general recovery scheme would not have to make use of cyclic autocorrelation evaluation of the received signals which is typical for most of cyclostationary beamformers (see [62] and references therein).

As a final step, transmitted symbol sequence estimation could be performed from the source estimates $\hat{u}_{g}\left(t_{k}\right) \forall g$ in (57) considering standard interpolation and symbol recovery techniques given a sufficiently large oversampling factor $U$. An interesting study could then compare the performance of such a procedure with that of a typical blind symbol recovery MIMO technique.

\section{Conclusion}

We presented a blind channel estimation algorithm for pulse-shaped communications signals exploiting only SOS and requiring no particular synchronization between transmitting and receiving antennas. The main contribution of the paper is the joint exploitation of the cyclostationary property of the received signals in a general asynchronous context as a means of creating a set of differential autocorrelation matrices suitable for a JD process or equivalent processing. This operation was shown to eliminate the need of noise power or statistical distribution estimation, and its effectiveness was confirmed in a broad set of simulation experiments.

The aim of the paper was also to provide clearer explanations on the main distinctions between synchronous and asynchronous signal modelings and their respective implications on the statistical distribution of the received signal vector. We also aimed at giving a more intuitive understanding of the traditional/classical and FIR-MIMO-based signal models showing how they equivalently relate to each other via different expressions of the received source vector. The two approaches have their own distinct advantages and disadvantages for the general BSS problem in a context of digital communications which are subject to further comparative analysis.

\section{APPENDix}

This section provides the main theoretical derivations of a source NAP such as defined in (17). Applications to common practical pulse shaping functions are also presented along with a discussion of some important follow-up results.

\section{A.1 NAP derivation}

A received source signal can be modeled similarly to (6) as an infinite pulse-shaped symbol stream of the form:

$$
u(t)=\sum_{m=-\infty}^{\infty} s_{m} p(t-m T),
$$

where $\left\{s_{m}\right\}_{m=-\infty}^{\infty}$ is an iid transmitted symbol sequence with zero-mean. Without loss of generality, no asynchronous delay is considered in this analysis since one may simply replace $t$ by $t-\tau_{0}$ to account for such a 
parameter. Assuming that the pulse shaping function $p(t)$ is real and deterministic, the signal autocorrelation is obtained as:

$$
\begin{aligned}
R_{u u}(t, \tau)= & E\left\{u(t) u^{*}(t+\tau)\right\} \\
= & E\left\{\left[\sum_{m=-\infty}^{\infty} s_{m} p(t-m T)\right] .\right. \\
& {\left.\left[\sum_{n=-\infty}^{\infty} s_{n}^{*} p(t-n T+\tau)\right]\right\} } \\
= & E\left\{\sum_{m=-\infty}^{\infty} \sum_{n=-\infty}^{\infty} s_{m} s_{n}^{*} p(t-m T) \cdot\right. \\
& p(t-n T+\tau)\} \\
= & \sum_{m=-\infty}^{\infty} \sum_{n=-\infty}^{\infty} E\left\{s_{m} s_{n}^{*}\right\} p(t-m T) p(t-n T+\tau) \\
= & \sigma_{\text {sym }}^{2} \sum_{m=-\infty}^{\infty} p(t-m T) p(t-m T+\tau),(\text { A.3 })
\end{aligned}
$$

where $\sigma_{\text {sym }}^{2}=E\left\{s_{m} s_{m}^{*}\right\}=\operatorname{Var}\left\{s_{m}\right\}$ is the symbol variance, or power. The NAP of a source $u(t)$ is obtained by the ratio of its autocorrelation $R_{u u}(t, \tau)$ to its symbol power $\sigma_{\text {sym, }}^{2}$ that is:

$\operatorname{NAP}(t, \tau)=\frac{R_{u u}(t, \tau)}{\sigma_{\text {sym }}^{2}}=\sum_{m=-\infty}^{\infty} p(t-m T) p(t-m T+\tau)$.

This general-form solution can be simplified considering that practical pulse-shaping is achieved by convolving a symbol stream with a filter impulse response of finite duration. More specifically, we have:

$$
p(t)=w(t) h(t)
$$

where $w(t)$ is a window function of finite duration and $h(t)$ represents an ideal filter impulse response. Eq. (A.4) can therefore be simplified by limiting the summation bounds to finite values such that:

$$
\operatorname{NAP}(t, \tau)=\sum_{m=b_{\ell}}^{b_{u}} p(t-m T) p(t-m T+\tau)
$$

where $b_{\ell}$ and $b_{u}$ depend on the nature of $p(t)$ as well as the values of $t$ and $\tau$ to which $\operatorname{NAP}(t, \tau)$ is evaluated. An examination of (A.4) shows that $\operatorname{NAP}(t, \tau)$ is periodic in $t$ with period $T$. Therefore, a complete characterisation is possible by restricting its evaluation to any time interval of width $T$. From the considerations of Fig. 14, which depicts an arbitrary pulse shaping function $p(t)$ of width $W=t_{2}-t_{1}$, evaluation of $\operatorname{NAP}(t, \tau)$ in the interval $t \in[0, T]$ for $\tau \geqslant 0$ leads to:

$$
\begin{gathered}
b_{\ell}=-\left\lfloor t_{2} / T\right\rfloor, \\
b_{u}=1-\left\lceil t_{1} / T\right\rceil,
\end{gathered}
$$

which are simply the appropriate bounds on $m$ implying that $p(t-m T) \geqslant 0$ in $t \in[0, T]$. Substituting (A.7)

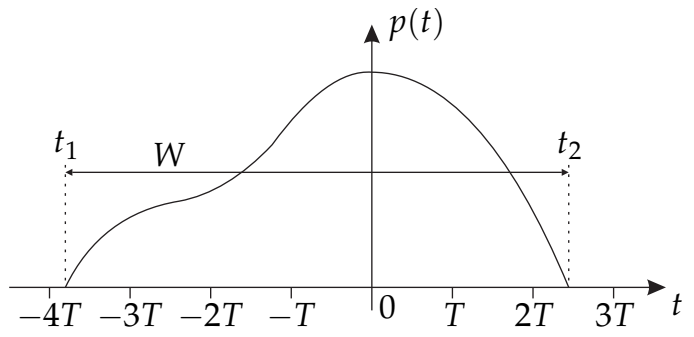

Figure 14. General pulse shaping function $p(t)$ spanning $W / T$ symbol periods.

into (A.6) yields:

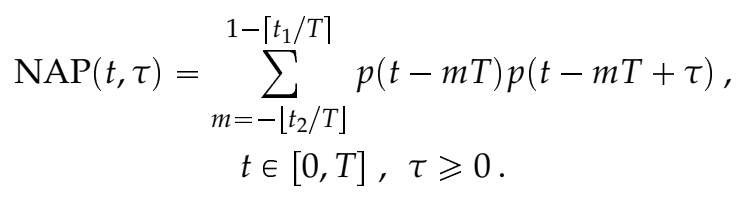

The latter equation has been used for the obtention of Fig. 4 using various combinations of $w(t)$ and $h(t)$ in (A.5). The NAP may also be obtained for any negative time lag considering the periodicity of $\operatorname{NAP}(t, \tau)$ and direct algebraic manipulations of (A.2). We have:

$$
\operatorname{NAP}(t,-\tau)=\operatorname{NAP}(t-\tau+k T, \tau),
$$

where $k$ is chosen such that $t-\tau+k T \in[0, T]$. Finally, let us recall that $p(t)$ in (A.1) represents a general received pulse function. Although ideal functions were considered in Section 5 and for the obtention of Fig. 4, practical calculations should be performed considering an effective waveform $\tilde{p}(t)$ taking into account channel propagation characteristics. For example, in the case of a linear time-invariant (LTI) channel,

$$
\tilde{p}(t)=p(t) * h_{\ell}(t)
$$

where $p(t)$ is the transmitted pulse and $h_{\ell}(t)$ is the equivalent low-pass channel impulse response [63].

\section{A.2 Raised cosine low-pass filter}

The raised cosine low-pass filter has an impulse response of the form:

$$
h(t)=\operatorname{sinc}(t / T) \frac{\cos (\pi \beta t / T)}{1-(2 \beta t / T)^{2}},
$$

where $\beta \in[0,1]$ is the roll-off factor specifying the filter excess bandwidth. Fig. 4 suggests that as $\beta \rightarrow 0$ and $W \rightarrow \infty, \operatorname{NAP}(t, \tau)$ tends toward a constant value, thus removing any potential autocorrelation diversity. In such conditions, the NAP evaluated from (A.4), (A.5) and (A.11) is given by:

$$
\operatorname{NAP}_{\left.R C\right|_{\beta=0}}(t, \tau)=\sum_{m=-\infty}^{\infty} \operatorname{sinc}(u-m) \operatorname{sinc}(u-m+v),
$$

where $u=t / T$ and $v=\tau / T$. This discrete autocorrelation function can be evaluated by taking the Fourier transform of both sides with respect to $v$. We have:

$$
\mathcal{F}_{v}\left\{\operatorname{NAP}_{\left.\mathrm{RC}\right|_{\beta=0}}(t, \tau)\right\}(\omega)
$$




$$
\begin{aligned}
& =\sum_{m=-\infty}^{\infty} \operatorname{sinc}(u-m) \mathcal{F}_{v}\{\operatorname{sinc}(u-m+v)\}(\omega) \\
& =\sum_{m=-\infty}^{\infty} \operatorname{sinc}(u-m)\left[\operatorname{rect}(\omega, 2 \pi) e^{-j \omega(m-u)}\right] \\
& =\operatorname{rect}(\omega, 2 \pi) e^{j \omega u} \sum_{m=-\infty}^{\infty} \operatorname{sinc}(u-m) e^{-j \omega m} \\
& \equiv \operatorname{rect}(\omega, 2 \pi) e^{j \omega u} S(u, \omega) .
\end{aligned}
$$

Function $S(u, \omega)$ corresponds to the discrete-time Fourier transform (DTFT) of $\operatorname{sinc}(u-m)$. From analogy with the time shift property ${ }^{14}$ and recalling that $\operatorname{sinc}(t)$ is an even function, we have:

$$
S(u, \omega)=\sum_{m=-\infty}^{\infty} \operatorname{sinc}(m) e^{-j \omega m} e^{-j \omega u}=e^{-j \omega u},
$$

since $\operatorname{sinc}(m)=0 \forall m \in \mathbb{Z}^{*}$. Substituting back into (A.13), we get:

$$
\mathcal{F}_{v}\left\{\left.\operatorname{NAP}_{\mathrm{RC}}\right|_{\beta=0}(t, \tau)\right\}(\omega)=\operatorname{rect}(\omega, 2 \pi),
$$

and therefore:

$$
\left.\operatorname{NAP}_{\mathrm{RC}}\right|_{\beta=0}(t, \tau)=\operatorname{sinc}(\tau / T) .
$$

A source NAP is hence independent of $t$ under ideal cardinal sine pulse shaping. This represents a worstcase scenario where autocorrelation techniques via sampling phase diversity cannot be exploited.

\section{A.3 Gaussian low-pass filter}

The Gaussian low-pass filter has an impulse response of the form:

$$
h(t)=\frac{\sqrt{\pi}}{\alpha} e^{-(\pi t / \alpha)^{2}} .
$$

Parameter $\alpha$ is related to the $3-\mathrm{dB}$ bandwidth $B$ of the filter [64] by:

$$
\alpha=\frac{\sqrt{\ln (2)} T}{\sqrt{2} B T}
$$

where $B T$ is the bandwidth-symbol time product. The impulse response extends to $\pm \infty$, but decays rapidly out of $t \in\left[\frac{-1}{2 B}, \frac{1}{2 B}\right]$. Therefore, calculations of Fig. 4 were performed considering a rectangular window of width $1 / B$ in (A.5). The NAP of a Gaussian pulse-shaped signal (unwindowed) is given by:

$$
\operatorname{NAP}_{\text {Gauss }}(t, \tau)=\frac{\pi}{\alpha^{2}} \sum_{m=-\infty}^{\infty} e^{-\frac{\pi^{2}}{\alpha^{2}}\left((t-m T)^{2}+(t-m T+\tau)^{2}\right)},
$$

where no equivalent closed-form solution could be found. However, as $\alpha \rightarrow \infty$, it is easily shown that $\operatorname{NAP}_{\text {Gauss }}(t, \tau) \rightarrow 0$, a tendency that can also be observed in Fig. 4.

\footnotetext{
${ }^{14}$ DTFT: $X(\omega)=\sum_{n=-\infty}^{\infty} x[n] e^{-j \omega n}$. Time shift property: $x[n-$ $\left.n_{0}\right] \leftrightarrow X(\omega) e^{-j \omega n_{0}}$.
}

\section{ACKNOWLEDGEMENT}

The authors would like to thank the reviewers and the dedicated personnel of JEC for their useful comments and suggestions.

\section{REFERENCES}

[1] A. Alexiou and M. Haardt, "Smart antenna technologies for future wireless systems: trends and challenges," IEEE Communications Magazine, vol. 42, no. 9, pp. 90-97, 2004.

[2] T. Do-Hong, Wideband direction of arrival estimation and wideband beamforming for smart antenna systems. Shaker Verlag GmbH, 2004.

[3] F. B. Gross, Smart antennas for wireless communications with MATLAB. McGraw-Hill, 2005.

[4] L. M. Correia, Mobile Broadband Multimedia Networks: Techniques, Models and Tools for 4G. Academic Press, 2006.

[5] R. Schmidt, "Multiple emitter location and signal parameter estimation," IEEE Transactions on Antennas and Propagation, vol. 34, no. 3, pp. 276-280, 1986.

[6] R. Roy and T. Kailath, "Esprit-estimation of signal parameters via rotational invariance techniques," IEEE Transactions on Acoustics, Speech and Signal Processing, vol. 37, no. 7, pp. 984-995, 1989.

[7] L. De Lathauwer, J. Castaing, and J.-F. Cardoso, "Fourthorder cumulant-based blind identification of underdetermined mixtures," IEEE Transactions on Signal Processing, vol. 55, no. 6, pp. 2965-2973, 2007.

[8] P. Comon, "Blind channel identification and extraction of more sources than sensors," Advanced Signal Processing Algorithms, Architectures, and Implementations VIII, vol. 3461, pp. 2-13, 1998.

[9] G. Sun, "MPSK signals modulation classification using sixth-order cumulants," in 3rd International Congress on Image and Signal Processing (CISP), 2010, vol. 9. IEEE, 2010, pp. 4404-4407.

[10] N. Yuen and B. Friedlander, "Doa estimation in multipath: an approach using fourth-order cumulants," IEEE Transactions on Signal Processing, vol. 45, no. 5, pp. 12531263, 1997.

[11] J. Krolik and D. Swingler, "Multiple broad-band source location using steered covariance matrices," IEEE Transactions on Acoustics Speech and Signal Processing, vol. 37, no. 10, pp. 1481-1494, 1989.

[12] H. Yu, J. Liu, Z. Huang, Y. Zhou, and X. Xu, "A new method for wideband doa estimation," in International Conference on Wireless Communications, Networking and Mobile Computing, WiCom 2007. IEEE, 2007, pp. 598601.

[13] A. Taleb, "An algorithm for the blind identification of $\mathrm{N}$ independent signals with 2 sensors," in Sixth International Symposium on Signal Processing and its Applications, vol. 1. IEEE, 2001, pp. 5-8.

[14] P. Comon and M. Rajih, "Blind identification of underdetermined mixtures based on the characteristic function," Signal Processing, vol. 86, no. 9, pp. 2271-2281, 2006.

[15] M. Rajih and P. Comon, "Blind identification of underdetermined mixtures based on the characteristic function: influence of the knowledge of source pdf's," in 1st IEEE International Workshop on Computational Advances in Multi-Sensor Adaptive Processing. IEEE, 2005, pp. 133136.

[16] J.-F. Cardoso and A. Souloumiac, "Blind beamforming for non-gaussian signals," in Radar and Signal Processing, IEE Proceedings F, vol. 140, no. 6. IET, 1993, pp. 362-370.

[17] A. Belouchrani, K. Abed-Meraim, J.-F. Cardoso, and E. Moulines, "A blind source separation technique us- 
ing second-order statistics," IEEE Transactions on Signal Processing, vol. 45, no. 2, pp. 434-444, 1997.

[18] Y. Rong, S. A. Vorobyov, A. B. Gershman, and N. D. Sidiropoulos, "Blind spatial signature estimation via time-varying user power loading and parallel factor analysis," IEEE Transactions on Signal Processing, vol. 53, no. 5, pp. 1697-1710, 2005.

[19] A. C. Tang, M. T. Sutherland, and C. J. McKinney, "Validation of SOBI components from high-density EEG," NeuroImage, vol. 25, no. 2, pp. 539-553, 2005.

[20] A. Yeredor, "TV-SOBI: An expansion of SOBI for linearly time-varying mixtures," in Proceedings of The 4th International Symposium on Independent Component Analysis and Blind Source Separation (ICA2003), 2003.

[21] — "Blind separation of gaussian sources via secondorder statistics with asymptotically optimal weighting," IEEE Signal Processing Letters, vol. 7, no. 7, pp. 197-200, 2000.

[22] F. J. Theis and Y. Inouye, "On the use of joint diagonalization in blind signal processing," in Proceedings, IEEE International Symposium on Circuits and Systems, ISCAS 2006. IEEE, 2006, pp. 4-pp.

[23] P. Tichavsky and A. Yeredor, "Fast approximate joint diagonalization incorporating weight matrices," IEEE Transactions on Signal Processing, vol. 57, no. 3, pp. 878891, 2009.

[24] D.-Z. Feng, H. Zhang, and W. X. Zheng, "Bi-iterative algorithm for extracting independent components from array signals," IEEE Transactions on Signal Processing, vol. 59, no. 8, pp. 3636-3646, 2011.

[25] X.-F. Xu, D.-Z. Feng, and W. X. Zheng, "An improved method for blind separation of complex-valued signals via joint diagonalization," in IEEE International Symposium on Circuits and Systems (ISCAS), 2011. IEEE, 2011, pp. 637-640.

[26] G. Chabriel and J. Barrere, "A direct algorithm for nonorthogonal approximate joint diagonalization," IEEE Transactions on Signal Processing, vol. 60, no. 1, pp. 39-47, 2012.

[27] A. Yeredor, "Non-orthogonal joint diagonalization in the least-squares sense with application in blind source separation," IEEE Transactions on Signal Processing, vol. 50, no. 7, pp. 1545-1553, 2002.

[28] R. Vollgraf and K. Obermayer, "Quadratic optimization for simultaneous matrix diagonalization," IEEE Transactions on Signal Processing, vol. 54, no. 9, pp. 3270-3278, 2006.

[29] A. Ziehe, P. Laskov, G. Nolte, and K.-R. Müller, "A fast algorithm for joint diagonalization with non-orthogonal transformations and its application to blind source separation," The Journal of Machine Learning Research, vol. 5, pp. 777-800, 2004

[30] S. Dégerine, "Sur la diagonalisation conjointe approchée par un critère des moindres carrés," in 18-ème Colloque sur le traitement du signal et des images, FRA, 2001. GRETSI, Groupe dÉtudes du Traitement du Signal et des Images, 2001.

[31] S. Choi and A. Cichocki, "Blind separation of nonstationary sources in noisy mixtures," Electronics Letters, vol. 36, no. 9, pp. 848-849, 2000.

[32] _ "Blind separation of nonstationary and temporally correlated sources from noisy mixtures," in Proceedings of the IEEE Signal Processing Society Workshop on Neural Networks for Signal Processing X., vol. 1. IEEE, 2000, pp. 405-414.

[33] K. Nordhausen, "On robustifying some second order blind source separation methods for nonstationary time series," Statistical Papers, pp. 1-16, 2012.

[34] M. Tsatsanis and C. Kweon, "Blind source separation of non-stationary sources using second-order statistics," in Conference Record of the Thirty-Second Asilomar Conference on Signals, Systems $\mathcal{E}$ Computers, 1998., vol. 2. IEEE, 1998, pp. $1574-1578$.

[35] D. G. Manolakis, V. K. Ingle, and S. M. Kogon, Statistical and adaptive signal processing: spectral estimation, signal modeling, adaptive filtering, and array processing. Artech House, 2005, vol. 46.

[36] E. Gonen and J. M. Mendel, "Applications of cumulants to array processing-Part III: Blind beamforming for coherent signals," IEEE Transactions on Signal Processing, vol. 45, no. 9, pp. 2252-2264, 1997.

[37] E. Gonen, J. M. Mendel, and M. C. Dogan, “Applications of cumulants to array processing-Part IV: Direction finding in coherent signals case," IEEE Transactions on Signal Processing, vol. 45, no. 9, pp. 2265-2276, 1997.

[38] A. Swindlehurst, "Synchronization and spatial signature estimation for multiple known co-channel signals," in 1995 Conference Record of the Twenty-Ninth Asilomar Conference on Signals, Systems and Computers, vol. 1. IEEE, 1995, pp. 398-402.

[39] S. Talwar, M. Viberg, and A. Paulraj, "Blind separation of synchronous co-channel digital signals using an antenna array-Part I: Algorithms," IEEE Transactions on Signal Processing, vol. 44, no. 5, pp. 1184-1197, 1996.

[40] S. Talwar and A. Paulraj, "Blind separation of synchronous co-channel digital signals using an antenna array-Part II: Performance analysis," IEEE Transactions on Signal Processing, vol. 45, no. 3, pp. 706-718, 1997.

[41] A. J. Paulraj and C. B. Papadias, "Space-time processing for wireless communications," IEEE Signal Processing Magazine, vol. 14, no. 6, pp. 49-83, 1997.

[42] P. McLane, "A residual intersymbol interference error bound for truncated-state viterbi detectors," IEEE Transactions on Information Theory, vol. 26, no. 5, pp. 548-553, 1980.

[43] B. Porat and B. Friedlander, "Direction finding algorithms based on high-order statistics," IEEE Transactions on Signal Processing, vol. 39, no. 9, pp. 2016-2024, 1991.

[44] R. Attux, R. Suyama, R. Ferrari, C. Junqueira, R. Krummenauer, P. Larzabal, and A. Lopes, "A clustering-based method for DOA estimation in wireless communications," 2007.

[45] F. Gu, H. Zhang, N. Li, and W. Lu, "Blind separation of multiple sequences from a single linear mixture using finite alphabet," in 2010 International Conference on Wireless Communications and Signal Processing (WCSP). IEEE, 2010, pp. 1-5.

[46] J. H. Manton and Y. Hua, "A randomised algorithm for improving source and channel estimates by exploiting the finite alphabet property," in Conference Record of the Thirty-Fourth Asilomar Conference on Signals, Systems and Computers, vol. 2. IEEE, 2000, pp. 1582-1585.

[47] J. D. Terry and D. B. Williams, "Convergence analysis of finite alphabet beamformers for digital cochannel signals," IEEE Transactions on Communications, vol. 51, no. 6, pp. 929-939, 2003

[48] K. I. Diamantaras, "A clustering approach for the blind separation of multiple finite alphabet sequences from a single linear mixture," Signal processing, vol. 86, no. 4, pp. 877-891, 2006.

[49] J. Camparo, R. Frueholz, and A. Dubin, "Demonstration of synchronization between two geosynchronous satellites without ground intervention," DTIC Document, Tech. Rep., 1996.

[50] S. Glisic, Advanced Wireless Communications: 4G Cognitive and Cooperative Broadband Technology. John Wiley \& Sons, 2007.

[51] T. Heikkinen and A. Hottinen, "Delay-differentiated scheduling in a fading channel," IEEE Transactions on Wireless Communications, vol. 7, no. 3, pp. 848-856, 2008.

[52] C. A. R. Fernandes, A. Kibangou, G. Favier, and J. C. Mota, "Identification of nonlinear mimo radio over fiber uplink channels," in Telecommunications Symposium, 2006 International. IEEE, 2006, pp. 213-218.

[53] T. Dubois, M. Crussiere, and M. Hélard, "On the use 
of time reversal for digital communications with nonimpulsive waveforms," in 4 th International Conference on Signal Processing and Communication Systems (ICSPCS). IEEE, 2010, pp. 1-6.

[54] T. S. Rappaport, Wireless communications: principles and practice. Prentice Hall, 2002.

[55] N. D. Sidiropoulos, G. B. Giannakis, and R. Bro, "Blind PARAFAC receivers for DS-CDMA systems," IEEE Transactions on Signal Processing, vol. 48, no. 3, pp. 810-823, 2000.

[56] W. A. Gardner, A. Napolitano, and L. Paura, "Cyclostationarity: Half a century of research," Signal processing, vol. 86, no. 4, pp. 639-697, 2006.

[57] J.-F. Cardoso, "On the performance of orthogonal source separation algorithms," in Proc. EUSIPCO, vol. 94. Edinburgh, UK, 1994, pp. 776-779.

[58] A. Yeredor, "On using exact joint diagonalization for noniterative approximate joint diagonalization," IEEE Signal Processing Letters, vol. 12, no. 9, pp. 645-648, 2005.

[59] A.-J. Van Der Veen, "Joint diagonalization via subspace fitting techniques," in Proceedings of the IEEE International Conference on Acoustics, Speech, and Signal Processing (ICASSP'01), vol. 5. IEEE, 2001, pp. 2773-2776.

[60] H. Akaike, "A new look at the statistical model identification," IEEE Transactions on Automatic Control, vol. 19, no. 6, pp. 716-723, 1974.

[61] J. Rissanen, "Modeling by shortest data description," Automatica, vol. 14 , no. 5, pp. 465-471, 1978.

[62] K.-L. Du and M. N. Swamy, "A class of adaptive cyclostationary beamforming algorithms," Circuits, Systems, and Signal Processing, vol. 27, no. 1, pp. 35-63, 2008.

[63] A. Goldsmith, Wireless Communications. Cambridge University Press, 2005.

[64] R. Staszewski and P. Balsara, All-Digital Frequency Synthesizer in Deep-Submicron CMOS. Wiley-Interscience, 2006.

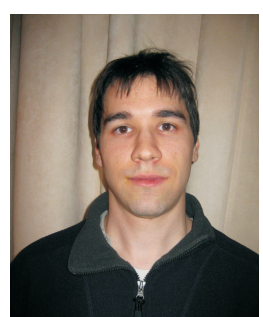

Emmanuel Racine was graduated in electromechanical engineering in 2007 from the Université du Québec en AbitibiTémiscamingue (UQAT), Canada. He afterward received his M.Sc. degree in 2009 at Université Laval, Canada, where he studied the problem of direction of arrival (DOA) estimation using an antenna array within the Laboratoire de Radiocommunications et Traitement du Signal (LRTS). He pursued his Ph.D. studies in the same subject, also at Université Laval, where he focused on exploiting intrinsic statistical signal properties for beamforming (BF) enhancement or general signal parameter estimation purposes. In particular, he developed algorithms based on higher-order statistics (HOS), nonlinear estimators and cyclostationary properties of communication signals.

Racine has contributed to the scientific community through publication of several journal and conference papers in his domain. He has also contributed to academic programs and university operations by working as teaching assistant and being tutor for a mentorship programme within the Ordre des ingénieurs du Québec (OIQ) in various disciplines.

Racine enjoys challenges and rigorous approaches to practical scientific problems. His main interest of research include signal processing and its applications, general electromagnetism and high frequency power system design.

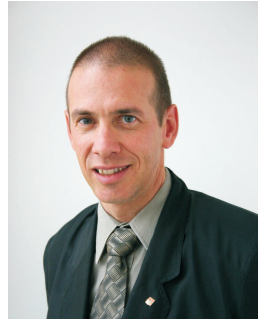

Dominic Grenier received the M.Sc and Ph.D. degrees in electrical engineering in 1985 and 1989, respectively, from the Université Laval, Quebec City, Canada.

From 1989 to 1990, he was a Postdoctoral Fellow in the radar division of the Defense Research Establishment in Ottawa (DREO) Canada. In 1990, he joined the Department of Electrical Engineering at Université Laval where he is currently a Full Professor since 2000. He was also co-editor for the Canadian Journal on Electrical and Computer Engineering during 6 years.

$\mathrm{He}$ is recognized by the undergraduate students in electrical and computer engineering at Université Laval as the electromagnetism and RF specialist. His excellence in teaching has resulted in "Best Teacher Award" directly from student's associations many times, the SUMMA-teaching award from Engineering Faculty and the Excellence-teaching award from Université Laval in 2010; finally he received a special teaching recognition award in 2012 from "Ordre des ingénieurs du Québec" (OIQ). He obtained in 2009 one special fellowship for teaching from the Quebec Minister for education.

His research interests include inverse synthetic aperture radar imaging (ISAR), signal array processing for high resolution direction of arrivals, information fusion for identification, and reflectometry probes design and signal processing. He has many collaborations with Development and Research for Defence Canada (DRDC-V) center, and with industries under grants or contracts.

Prof. Grenier has 35 publications in refereed journals and 75 more in conference proceedings. In addition, more than 40 graduate students completed their thesis under his direction since 1992.

Prof. Grenier is a registered professional engineer in the Province of Quebec (OIQ), Canada. 